\title{
Probing the gluon Sivers function through direct photon production at RHIC
}

\author{
Rohini M. Godbole, ${ }^{1,2,4, *}$ Abhiram Kaushik, ${ }^{1, \dagger}$ Anuradha Misra, ${ }^{3, *}$ and Siddhesh Padval ${ }^{3,8}$ \\ ${ }^{1}$ Centre for High Energy Physics, Indian Institute of Science, Bangalore 560012, India \\ ${ }^{2}$ Institute of Physics and Astronomy, University of Amsterdam, \\ Science Park 904, 1098 XH Amsterdam, The Netherlands \\ ${ }^{3}$ Department of Physics, University of Mumbai, Mumbai 400098, India \\ ${ }^{4}$ Nikhef, Science Park, 1098 XG Amsterdam, The Netherlands
}

(Received 22 October 2018; published 3 January 2019)

\begin{abstract}
We study the production of prompt photons at the RHIC in the context of a generalized parton model framework, with a view to obtain information on the gluon Sivers function (GSF). At RHIC energy $(\sqrt{s}=200 \mathrm{GeV})$, the Compton process $g q \rightarrow \gamma q$ contributes significantly to the production of direct photons at midrapidity and dominates it in the negative (backward) rapidity region. We find that for direct photons, asymmetries of up to $10 \%$ are allowed by a maximal gluon Sivers function. However, the asymmetry obtained using existing fits of the GSF available in the literature is negligible. We also estimate the impact that photons produced via fragmentation can have on the signal and find that their inclusion can dilute the asymmetry by between $10 \%$ and $50 \%$ of the direct photon value. Finally, using the color-gauge invariant generalized parton model (CGI-GPM) approach, we consider the effects of initial-state and finalstate interactions which can affect the universality of the Sivers functions in different processes. We find that the inclusion of these effects leads to the size of the gluon contributions being roughly halved. However, in the backward region which we are interested in, the sizes of the quark contributions are suppressed even further, leading to increased dominance of the gluon contributions.
\end{abstract}

DOI: 10.1103/PhysRevD.99.014003

\section{INTRODUCTION}

Transverse single-spin asymmetries (SSA) can provide information on the three-dimensional structure of hadrons. They have hence been a subject of great interest in recent times. In the past few years, a large amount of data on SSAs have become available in a wide variety of processes such as semi-inclusive deep-inelastic scattering, hadroproduction of light and heavy mesons (see Refs. [1,2] for reviews of experimental data on the subject) and most recently in Drell-Yan [3]. One of the theoretical approaches used to describe these asymmetries is TMD factorization [4-7]. In this approach, factorization in terms of transversemomentum-dependent parton distribution functions (TMDPDF) and fragmentation functions (TMD-FF) is assumed. These functions depend on the transverse momentum of the

\footnotetext{
rohini@iisc.ac.in

†abhiramk@iisc.ac.in

*misra@physics.mu.ac.in

§siddhesh.padval@physics.mu.ac.in
}

Published by the American Physical Society under the terms of the Creative Commons Attribution 4.0 International license. Further distribution of this work must maintain attribution to the author(s) and the published article's title, journal citation, and DOI. Funded by SCOAP . parton in addition to the light-cone momentum fraction; i.e., they are of the form $f_{i / h}(x, \boldsymbol{k}, Q)$ and $D_{h / i}(z, \boldsymbol{k}, Q)$ respectively. This is in contrast to the commonly used collinear PDFs and FFs, which depend only on the light-cone momentum fraction as the transverse momentum of the parton is integrated over. So far, TMD factorization has been demonstrated only for processes which have two scales: a hard, high energy scale such as the virtuality of the photon in the Drell-Yan process and a relatively soft scale of the order of $\Lambda_{\mathrm{QCD}}$, such as the transverse momentum of the Drell-Yan lepton pair. In the TMD approach, one of the main TMDs that can lead to a SSA is the Sivers distribution [8,9]. This encodes the correlation between the azimuthal anisotropy in the distribution of an unpolarized parton and the spin of its parent hadron. This anisotropy in the parton's transversemomentum distribution can lead to an azimuthal anisotropy in the distribution of the inclusive final state, i.e., a SSA.

Though TMD factorization has not been formally established for hard single-scale processes such as $p^{\uparrow} p \rightarrow$ $h+X$ and $p^{\uparrow} p \rightarrow \gamma+X$, an effective description of SSAs in such processes in terms of the TMDs-under the assumption of factorization and universality-has been phenomenologically successful [10-15]. This effective description is commonly referred to in the literature as the generalized parton model (GPM). 
Recently a modification of the generalized parton model has been proposed in which the process dependence of the Sivers function is taken into account. In this approach, known as the color-gauge invariant generalized parton model (CGI-GPM) [16-18], the process-dependent initialstate interactions (ISIs) and final-state interactions (FSIs) are treated using one-gluon exchange approximation. These interactions provide the complex phase necessary for the SSA. The process dependence of the Sivers functions, which arises from these interactions, is then shifted onto appropriately defined "modified" partonic cross sections and the Sivers functions can still be treated as universal. This approach was first proposed in Ref. [16], where they considered quark Sivers functions (QSF), and has recently been extended to include gluon Sivers functions in Ref. [19]. The process dependence of the Sivers function (and in general T-odd functions) was earlier studied in the context of two-scale processes in Refs. [20,21].

While the quark Sivers functions have been widely studied over the years, the gluon Sivers function (GSF) still remains poorly measured. An indirect estimate of the gluon Sivers function was obtained using a GPM framework in Ref. [22] where the authors fit the gluon Sivers function to midrapidity data on SSA in $\pi^{0}$ production at the RHIC. In the analysis, the quark contribution to the SSA was calculated using QSFs as extracted from semi-inclusive deep inelastic scattering (SIDIS) data. The small and positive GSF fits obtained by the analysis predicted asymmetries much smaller than allowed by the positivity bound on the GSF. The said bound restricts the GSF to be less than twice the unpolarized TMD gluon distribution. Further, a recent study of large- $p_{T}$ hadron pair production in COMPASS indicates a substantial, negative gluon Sivers asymmetry but with large errors and hence consistent with zero at the 2.5 sigma level for a proton target [23]. Large- $p_{T}$ hadron pairs are produced in this process through photongluon fusion, a process which gives direct access to the gluon content of the proton. The differences between these two results, though of limited statistical significance, make it clear that the GSF needs to be studied in more detail and with unambiguous probes.

More direct probes of the GSF are thus needed. Closed and open heavy-flavor production offers such probes. A GPM study of open charm production as a probe of the GSF was proposed in Ref. [24] for the process $p^{\uparrow} p \rightarrow$ $D^{0}+X$. Therein they considered two extreme scenarios for the GSF: zero and saturated. By "saturated" we mean the Sivers function with its positivity bound of twice the unpolarized TMD, i.e., $\left|\Delta^{N} f_{i / p^{\uparrow}}\left(x, \mathbf{k}_{\perp}\right)\right| / 2 f_{i / p}\left(x, \mathbf{k}_{\perp}\right) \leq 1$, saturated for all values of $x$. Their study indicated that an observation of SSA for this process at the RHIC can give a direct indication of a nonzero gluon Sivers function. Further in Ref. [25] we calculated the SSA for the same process (open charm hadroproduction) using the fits of Ref. [22] and found that these fits predict sizable, measurable asymmetries. In Ref. [26], we proposed the low-virtuality leptoproduction of open charm and studied it in the context of the GPM framework. Unlike hadroproduction, this process does not involve any contributions from quarks and hence can probe the gluon Sivers function effectively. In this case too we found results similar to those for the hadroproduction of open charm. For the kinematics of COMPASS and a future electron-ion collider (EIC), the fits of Ref. [22] gave sizable and distinct asymmetries. Further we found that the asymmetry was well preserved in the kinematics of the muons decaying from the $D$-meson.

Apart from the production of open charm, probes involving the production of closed charm, i.e., $J / \psi$, can also give direct access to the gluon content of the proton. The low-virtuality leptoproduction of closed charm, i.e., $J / \psi$, was proposed as a probe of the GSF in Refs. [27-29] and the hadroproduction of closed charm was studied in Refs. [19,30]. Recently, the PHENIX Collaboration at the RHIC has measured the SSA in the production of $J / \psi$ in $p^{\uparrow} p$ collisions [31]. They find that the data indicate a positive asymmetry at the two-standard-deviation level in the $x_{F}<0$ region.

In this work, using both the GPM and CGI-GPM approaches, we study the hadroproduction of prompt photons as a possible probe of the gluon Sivers function. By prompt photons we mean both the direct photons which are created in the hard process and fragmentation photons which are created by fragmentation of outgoing partons from the hard process. At leading order (LO) in $\alpha_{s}$, direct photons are produced through the fundamental 2-to-2 hard scattering subprocesses, $g q \rightarrow \gamma q$ and $q \bar{q} \rightarrow \gamma+g$. The first of these subprocesses, the QCD Compton process, dominates in $p p$ collisions. Indeed direct photon data from fixed target experiments were used in early global fits of the collinear PDFs in unpolarized protons to constrain the gluon component [32-34]. At the RHIC, the study of prompt photon production in the midrapidity and backwards rapidity regions should give clean and direct access to the gluon content of the polarized proton, due to the dominance of the QCD Compton process. Since the photon is produced in the hard process and is colorless, the probe is unaffected by theoretical uncertainties related to hadronization or final-state interactions. For this reason, SSA in the production of direct photons in the backward region was first proposed as a probe of the gluon Sivers function by Schmidt, Soffer and Yang (SSY) [35]. They suggested in general the large $-P_{T}$ region in the backward hemisphere. The SSA in this process has also been studied in the context of an alternative mechanism in the color glass condensate formalism and found to be zero [36].

In this work, we follow up on the work of SSY and consider estimates of the asymmetry in the production of direct photons in the backwards, i.e., negative rapidity region. In their work, SSY neglected the partonic transverse momenta in the hard part. However it is known that partonic 
transverse momenta cannot be neglected in the hard part. They lead to an important consequence, viz. a suppression of the SSA in the backward region relative to the forward region, as was found for the case of open-charm production in Ref. [24]. Here, we include the effect of partonic transverse momenta in the hard process. We find that after the inclusion of these effects, the dominance of the gluon contribution in the backward region continues even though the SSAs are suppressed. In this work, following our earlier studies of the GSF in open charm production $[25,26]$, we consider estimates for the asymmetry obtained using saturated quark and gluon Sivers functions. The use of saturated QSFs and GSF gives the upper bound on the possible asymmetry and further allows us to study the general kinematic dependencies of the asymmetry and the relative importances of the quark and gluon contributions to the asymmetry. It also allows us to assess the sensitivity of the probe to the uncertainties in our current knowledge of the collinear PDFs. Further we also consider the contribution to the asymmetry from photons produced via the fragmentation of partons. While the contribution to the signal and hence the asymmetry coming from the fragmentation component can be reduced to an extent by applying a photon isolation requirement, it cannot be completely eliminated. Hence it is important to study the impact that it can have on the asymmetry and hence on this probe of the GSF. Therefore, we also consider the asymmetry in the inclusive (direct as well as fragmentation) photons and study how it differs from the direct photon asymmetry.

We then consider existing fits of the QSFs and the GSF to give predictions for the expected asymmetry. We consider two fits of the QSFs [37,38], both of which have been obtained by fitting to data on semi-inclusive deep-inelastic scattering. Associated with these two QSF sets are two fits of the GSF in Ref. [22] which, as mentioned earlier, were obtained by fitting to the data on SSA in midrapidity $\pi^{0}$ production at the RHIC. Further we also consider indirect bounds on the gluon Sivers function based on the Burkardt sum rule (BSR) [39]. The BSR is the requirement that the net transverse momenta of all partons in a transversely polarized proton must vanish. The latest fits of the QSFs from Ref. [38] allow a gluon transverse momentum in the range $-10 \leq\left\langle k_{\perp g}\right\rangle \leq 48 \mathrm{MeV}$. This constraint on the allowed transverse momentum can be used to constrain the size of the gluon contribution to the asymmetry. We plot the asymmetry values allowed by the BSR constraint, along with the predictions from the fits.

Finally we study how the results of the above analysis are affected when we take into account the effects of initialand final-state interactions using the CGI-GPM approach. We do so for both the direct and the fragmentation contributions to the asymmetry.

This paper is organized as follows. In Sec. II, we give expressions for the relevant quantities in the GPM framework. In Sec. III, we present the CGI-GPM formalism and give the modified hard part for the relevant processes. In Sec. IV we present the parametrization of the various TMDs in the analysis as well as details of the QSF and the GSF parameters used. Finally, in Sec. V we present estimates of the asymmetry in both the GPM and CGIGPM frameworks.

\section{PROMPT PHOTON PRODUCTION IN THE GPM FORMALISM}

Prompt photons can be produced either in the hard scattering or through the fragmentation of a final-state parton into a photon. We refer to the former as "direct" photons and the latter as "fragmentation" photons. At leading order $\mathcal{O}\left(\alpha_{s} \alpha_{\mathrm{em}}\right)$, direct photons are produced through the QCD Compton process, $g q \rightarrow \gamma q$, and quark-antiquark annihilation into a photon and a gluon, $q \bar{q} \rightarrow \gamma g$. Of these $g q \rightarrow \gamma q$ dominates at RHIC energy and hence the production of direct photons can give direct access to the gluon content of the proton.

Fragmentation photons can be produced at leading order, $\mathcal{O}\left(\alpha_{s}^{2} \alpha_{\mathrm{em}}\right)$, through the standard 2-to-2 QCD parton scattering processes with the final-state parton fragmenting into a photon. Here, unlike direct photon production, the partonic subprocess is at $\mathcal{O}\left(\alpha_{s}^{2}\right)$ instead of $\mathcal{O}\left(\alpha_{s} \alpha_{\mathrm{em}}\right)$. Though this is naively higher order in $\alpha_{s}$ as compared to direct photon production, the parton-to-photon fragmentation functions (FFs) grow logarithmically with $Q^{2}$, making them effectively of order $1 / \alpha_{s}$. This logarithmic growth of the parton-to-photon FFs makes the production of fragmentation photons effectively at the same leading order $\mathcal{O}\left(\alpha_{s} \alpha_{\mathrm{em}}\right)$ as the production of direct photons. Though the fragmentation photon contribution can be discriminated by applying a photon isolation requirement, it can never be completely eliminated. Therefore it is important to consider the effect of fragmentation photons on the observed asymmetry in the consideration of SSAs in an inclusive signal. An illustration of both mechanisms of prompt photon production is given in Fig. 1.

In this work, we are concerned with the single-spin asymmetry in the hadroproduction of prompt photons,

$$
A_{N}=\frac{d \sigma^{\uparrow}-d \sigma^{\downarrow}}{d \sigma^{\uparrow}+d \sigma^{\downarrow}}
$$

where $d \sigma^{\uparrow(\downarrow)}$ is the invariant differential cross section for the process $p^{\uparrow(\downarrow)} p \rightarrow \gamma+X$ with the spin of the transversely polarized proton being aligned in the $\uparrow(\downarrow)$ direction with respect to the production plane. Here, $\uparrow$ would be the $+Y$ direction in a frame where the polarized proton is moving along the $+Z$ direction and the photon is produced in the $X Z$ plane.

In the following, we give the expressions for the denominator and numerator of Eq. (1) for the case of both direct photons and fragmentation photons. 

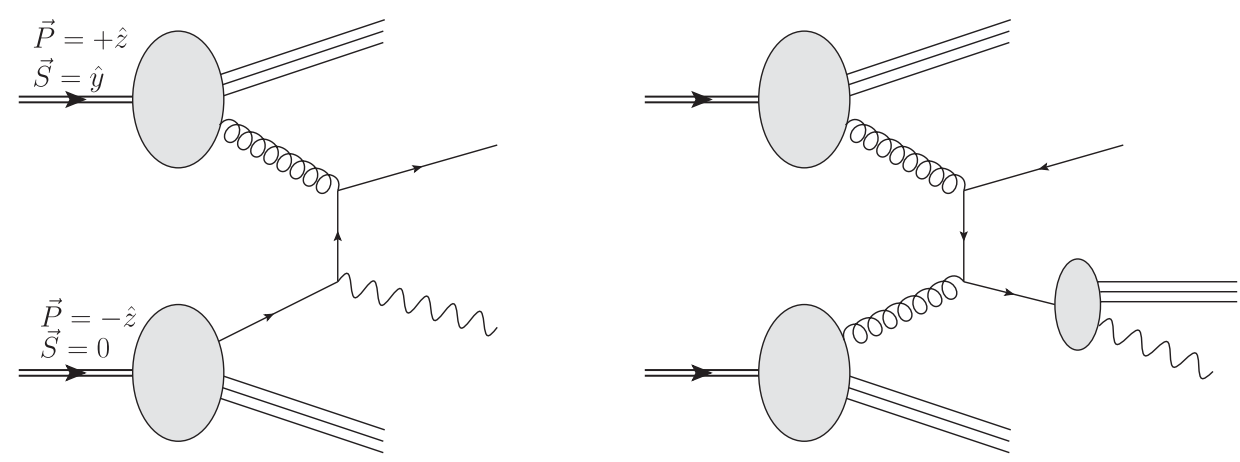

FIG. 1. Representative diagrams for prompt photon production at the hard scattering (left) and in the fragmentation of a final-state parton (right), in hadron-hadron collisions. We consider one of the hadrons to be a proton moving in the $+Z$ direction, with a polarization along the $+\mathrm{Y}$ axis. The other hadron is an unpolarized proton moving along the $-\mathrm{Z}$ direction.

\section{A. Direct photon production}

For direct photons, we can write the denominator and numerator of Eq. (1) as

$$
\begin{aligned}
d \sigma^{\uparrow}+d \sigma^{\downarrow} & =\frac{E_{\gamma} d \sigma^{p^{\uparrow} p \rightarrow \gamma X}}{d^{3} \boldsymbol{p}_{\gamma}}+\frac{E_{\gamma} d \sigma^{p^{\downarrow} p \rightarrow \gamma X}}{d^{3} \boldsymbol{p}_{\gamma}} \\
& =2 \sum_{a, b=g, q, \bar{q}} \int d x_{a} d^{2} \boldsymbol{k}_{\perp a} d x_{b} d^{2} \boldsymbol{k}_{\perp b} f_{a / p}\left(x_{a}, k_{\perp a}\right) f_{b / p}\left(x_{b}, k_{\perp b}\right) \frac{\hat{s}}{x_{a} x_{b} s} \frac{d \hat{\sigma}^{a b \rightarrow \gamma d}}{d \hat{t}} \frac{\hat{s}}{\pi} \delta(\hat{s}+\hat{t}+\hat{u})
\end{aligned}
$$

and

$$
\begin{aligned}
d \sigma^{\uparrow}-d \sigma^{\downarrow} & =\frac{E_{\gamma} d \sigma^{p^{\uparrow} p \rightarrow \gamma X}}{d^{3} \boldsymbol{p}_{\gamma}}-\frac{E_{\gamma} d \sigma^{p^{\downarrow} p \rightarrow \gamma X}}{d^{3} \boldsymbol{p}_{\gamma}} \\
& =\sum_{a, b=g, q, \bar{q}} \int d x_{a} d^{2} \boldsymbol{k}_{\perp a} d x_{b} d^{2} \boldsymbol{k}_{\perp b} \Delta^{N} f_{a / p^{\uparrow}}\left(x_{a}, \boldsymbol{k}_{\perp a}\right) f_{b / p}\left(x_{b}, k_{\perp b}\right) \frac{\hat{s}}{x_{a} x_{b} s} \frac{d \hat{\sigma}^{a b \rightarrow \gamma d}}{d \hat{t}} \frac{\hat{s}}{\pi} \delta(\hat{s}+\hat{t}+\hat{u}) .
\end{aligned}
$$

In the above expressions, $x_{a}$ and $x_{b}$ are the light-cone momentum fractions of the incoming partons of the polarized and unpolarized proton respectively. $\boldsymbol{k}_{\perp a}$ and $\boldsymbol{k}_{\perp b}$ are the transverse momenta of the partons $a$ and $b . \hat{s}=\left(p_{a}+p_{b}\right)^{2}$, $\hat{t}=\left(p_{\gamma}-p_{a}\right)^{2}$ and $\hat{u}=\left(p_{\gamma}-p_{b}\right)^{2}$ are the Mandelstam variables for the relevant subprocesses: the QCD Compton scattering process $g q \rightarrow \gamma q$, as well as $q \bar{q} \rightarrow \gamma g$.

The expressions $\Delta^{N} f_{i / p^{\uparrow}}\left(x, \mathbf{k}_{\perp}\right)$ and $f_{i / p}\left(x, \mathbf{k}_{\perp}\right)$ are the Sivers function and unpolarized TMD for parton $i$, respectively. The functional forms used for these two distributions are given in Sec. IV.

The Sivers function $\Delta^{N} f_{i / p^{\uparrow}}\left(x, k_{\perp} ; Q\right)$ describes the azimuthal anisotropy in the transverse-momentum distribution of an unpolarized parton, in a transversely polarized hadron,

$$
\begin{aligned}
& f_{i / h^{\uparrow}}\left(x, \mathbf{k}_{\perp}, \mathbf{S} ; Q\right) \\
& \quad=f_{i / h}\left(x, k_{\perp} ; Q\right)+\frac{1}{2} \Delta^{N} f_{i / h^{\uparrow}}\left(x, k_{\perp} ; Q\right) \frac{\epsilon_{a b} k_{\perp}^{a} S^{b}}{k_{\perp}} \\
& \quad=f_{i / h}\left(x, k_{\perp} ; Q\right)+\frac{1}{2} \Delta^{N} f_{i / h^{\uparrow}}\left(x, k_{\perp} ; Q\right) \cos \phi_{\perp}
\end{aligned}
$$

where $\mathbf{k}_{\perp}=k_{\perp}\left(\cos \phi_{\perp}, \sin \phi_{\perp}\right)$. Another notation, $f_{1 T}^{\perp i}$, is also commonly used for the Sivers function and is related to $\Delta^{N} f_{i / h^{\uparrow}}$ by

$$
\Delta^{N} f_{i / h^{\uparrow}}\left(x, k_{\perp}\right)=-2 \frac{k_{\perp}}{M_{h}} f_{1 T}^{\perp i}\left(x, k_{\perp}\right),
$$

where $M_{h}$ is the mass of the hadron, which in this case is the proton. We will use this notation when discussing the CGI-GPM formalism in Sec. III.

The partonic cross sections can be written as

$$
\frac{d \hat{\sigma}^{a b \rightarrow \gamma d}}{d \hat{t}}=\frac{\pi \alpha_{s} \alpha_{\mathrm{em}}}{\hat{s}^{2}} H_{a b \rightarrow \gamma d}^{U},
$$

with the hard parts for the two subprocesses given by

$$
H_{g q \rightarrow \gamma q}^{U}=-\frac{e_{q}^{2}}{3}\left[\frac{\hat{u}}{\hat{s}}+\frac{\hat{s}}{\hat{u}}\right], \quad H_{q \bar{q} \rightarrow \gamma g}^{U}=\frac{8}{9} e_{q}^{2}\left[\frac{\hat{u}}{\hat{t}}+\frac{\hat{t}}{\hat{u}}\right] .
$$

The on-shell condition $\hat{s}+\hat{t}+\hat{u}=0$ can be used to fix one of the integration variables, in this case, $x_{b}$. 


\section{B. Photons from fragmentation of quarks}

For photons that are produced in the fragmentation of quarks, there can be two TMDs that contribute towards a SSA: the Sivers function and the Collins function [40]. Here we consider only the Sivers function. A model calculation of the quark-to-photon Collins function has shown that the contribution to the asymmetry from the Collins function is negligible [41]. Following the formalism used in single-inclusive hadron productions [14,25], the denominator and numerator of Eq. (1) can be written as

$$
\begin{aligned}
d \sigma^{\uparrow}+d \sigma^{\downarrow}= & \frac{E_{\gamma} d \sigma^{p^{\uparrow} p \rightarrow \gamma X}}{d^{3} \boldsymbol{p}_{\gamma}}+\frac{E_{\gamma} d \sigma^{p^{\downarrow} p \rightarrow \gamma X}}{d^{3} \boldsymbol{p}_{\gamma}} \\
= & 2 \sum_{a, b=g, q, \bar{q}} \int d x_{a} d^{2} \boldsymbol{k}_{\perp a} d x_{b} d^{2} \boldsymbol{k}_{\perp b} d z d^{3} \boldsymbol{k}_{\gamma} \delta\left(\boldsymbol{k}_{\gamma}, \hat{\boldsymbol{p}}_{q}\right) f_{a / p}\left(x_{a}, k_{\perp a}\right) f_{b / p}\left(x_{b}, k_{\perp b}\right) \\
& \times \frac{\hat{s}}{x_{a} x_{b} s} \frac{d \hat{\sigma}^{a b \rightarrow c d}}{d \hat{t}} \frac{\hat{s}}{\pi} \delta(\hat{s}+\hat{t}+\hat{u}) \frac{1}{z^{2}} J\left(z,\left|\boldsymbol{k}_{\gamma}\right|\right) D_{\gamma / q}\left(z, \boldsymbol{k}_{\gamma}\right)
\end{aligned}
$$

and

$$
\begin{aligned}
d \sigma^{\uparrow}-d \sigma^{\downarrow}= & \frac{E_{\gamma} d \sigma^{p^{\uparrow} p \rightarrow \gamma X}}{d^{3} \boldsymbol{p}_{\gamma}}-\frac{E_{\gamma} d \sigma^{p^{\downarrow} p \rightarrow \gamma X}}{d^{3} \boldsymbol{p}_{\gamma}} \\
= & \sum_{a, b=g, q, \bar{q}} \int d x_{a} d^{2} \boldsymbol{k}_{\perp a} d x_{b} d^{2} \boldsymbol{k}_{\perp b} d z d^{3} \boldsymbol{k}_{\gamma} \delta\left(\boldsymbol{k}_{\gamma} \cdot \hat{\boldsymbol{p}}_{q}\right) \Delta^{N} f_{a / p^{\uparrow}}\left(x_{a}, k_{\perp a}\right) f_{b / p}\left(x_{b}, k_{\perp b}\right) \\
& \times \frac{\hat{s}}{x_{a} x_{b} s} \frac{d \hat{\sigma}^{a b \rightarrow c d}}{d \hat{t}} \frac{\hat{s}}{\pi} \delta(\hat{s}+\hat{t}+\hat{u}) \frac{1}{z^{2}} J\left(z,\left|\boldsymbol{k}_{\gamma}\right|\right) D_{\gamma / q}\left(z, \boldsymbol{k}_{\gamma}\right) .
\end{aligned}
$$

In the above expressions $D_{\gamma / q}\left(z, \boldsymbol{k}_{\gamma}\right)$ is the TMD fragmentation function describing the fragmentation of the quark $q$ into a photon carrying a light-cone momentum fraction $z=p_{\gamma}^{+} / p_{q}^{+}$, and a transverse momentum $\boldsymbol{k}_{\gamma}$ with respect to the fragmenting quark direction. $J\left(z,\left|\boldsymbol{k}_{\gamma}\right|\right)$ is the Jacobian factor connecting the phase space of parton $c$ to the phase space of the photon. It is given by

$$
J\left(z,\left|\boldsymbol{k}_{\gamma}\right|\right)=\frac{\left(E_{\gamma}+\sqrt{E_{\gamma}^{2}-k_{\gamma}^{2}}\right)^{2}}{4\left(E_{\gamma}^{2}-k_{\gamma}^{2}\right)} .
$$

The delta function $\delta\left(\boldsymbol{k}_{\gamma} \cdot \hat{\boldsymbol{p}}_{q}\right)$ in Eqs. (9) and (10) confines the integration region for $\boldsymbol{k}_{\gamma}$ to the two-dimensional plane perpendicular to the direction of the fragmenting quark $\hat{\boldsymbol{p}}_{c}$, i.e.,

$$
\int d^{3} \boldsymbol{k}_{\gamma} \delta\left(\boldsymbol{k}_{\gamma} \cdot \hat{\boldsymbol{p}}_{c}\right) D_{\gamma / c}\left(z, \boldsymbol{k}_{\gamma}\right) \ldots=\int d^{2} \boldsymbol{k}_{\perp \gamma} D_{D / c}\left(z, \boldsymbol{k}_{\perp \gamma}\right) \ldots
$$

where $\boldsymbol{k}_{\perp \gamma}$ represents values of transverse momenta on the allowed plane. For photon production via fragmentation, at LO the partonic cross section is of order $\alpha_{s}^{2}$,

$$
\frac{d \hat{\sigma}^{a b \rightarrow c d}}{d \hat{t}}=\frac{\pi \alpha_{s}^{2}}{\hat{s}^{2}} H_{a b \rightarrow c d}^{U},
$$

and the relevant hard parts can be found, for instance, in Ref. [42].

The details of the treatment of parton kinematics for both direct and fragmentation processes are presented in the Appendix.

\section{THE CGI-GPM FORMALISM}

In the generalized parton model it is assumed that all the transverse-momentum-dependent densities are universal. For instance, one can use a quark Sivers function fitted to SIDIS data to calculate asymmetries in hadroproduction processes [22]. Similarly the GSF fitted to data on pion hadroproduction can be used to calculate the asymmetry in $J / \psi$ production [30]. However, different processes - take, e.g., SIDIS and Drell-Yan - can have different initial- and final-state interactions between the active partons and the spectators from the polarized proton. For instance, in SIDIS, the scattered quark can exchange soft gluons with the remnant of the proton. This would be a final-state interaction. In the case of Drell-Yan, the incoming quark from the unpolarized proton can exchange soft gluons with the transversely polarized proton. This would be an initialstate interaction. These interactions can affect the universality of the TMD densities. The effects of these different ISI/FSIs can be understood by looking at the different Wilson line configurations that are required to render the operator definition of the Sivers function gauge invariant. 


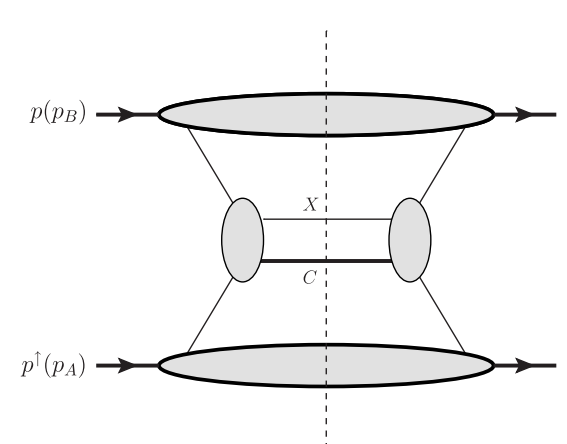

(a)

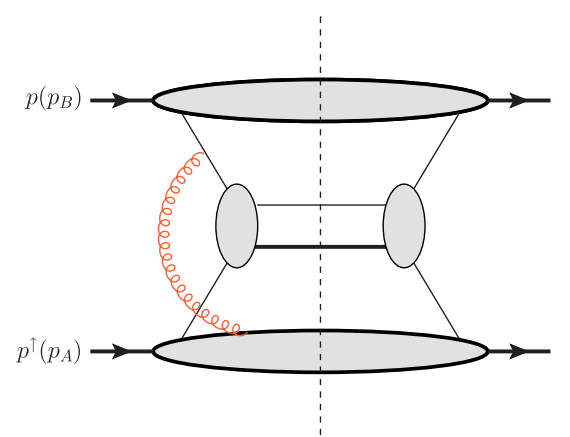

(b)

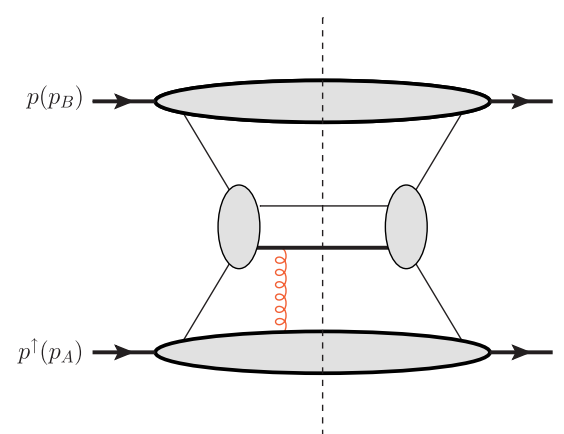

(c)

FIG. 2. LO diagrams for $A^{\uparrow}+B \rightarrow C+X$ in (a) GPM, (b) CGI-GPM with initial-state interactions and (c) final-state interactions. The eikonal soft gluon, shown in red, does not affect the kinematics of the process but only changes the color flow.

In the color-gauge invariant GPM formalism, these ISIs and FSIs are treated at the one-gluon exchange level, i.e., by expanding the Wilson lines to leading order in the coupling constant, $g_{s}$. To illustrate this, in Fig. 2, we show the diagrams for a process of the form $A^{\uparrow}+B \rightarrow C+X$ in (a) the standard GPM framework, and in the CGI-GPM framework with (b) the initial-state interactions and (c) the final-state interactions, both at the one-gluon exchange level. This approach was first proposed in Ref. [16] where the effects of the ISI/FSIs were calculated for the various quark-initiated subprocesses (subprocesses with a quark from the polarized proton) involved in $p^{\uparrow} p \rightarrow \pi+X$. They used their formalism to reproduce the expected sign flip between SIDIS and Drell-Yan Sivers asymmetries.

In order to obtain the asymmetry in the CGI-GPM formalism, we need to take into account the effects of the ISIs and FSIs. For processes that probe the QSFs, this is done by making the following substitutions in Eqs. (3) and (9):

$f_{1 T}^{\perp q} H_{q b \rightarrow c d}^{U} \equiv f_{1 T}^{\perp q} \sum_{i, j} \mathcal{A}_{i}^{*} \mathcal{A}_{j} \rightarrow \sum_{i, j} \frac{C_{I}^{i j}+C_{F_{c}}^{i j}}{C_{U}^{i j}} f_{1 T}^{\perp q} \mathcal{A}_{i}^{*} \mathcal{A}_{j}$.

Note that we have used an alternative notation for the Sivers function, which is related to the one in Eqs. (4) and (10) by $f_{1 T}^{\perp q}=-M_{p} \Delta^{N} f_{i / p^{\uparrow}} / 2 k_{\perp i}$. The above expression has various terms which we will explain now: the $\mathcal{A}_{i}$ are the amplitudes for the different channels that contribute to the subprocess $q b \rightarrow c d$. Here, $q$ corresponds to the quark from the polarized proton. On the right-hand side, $C_{U}^{i j}$ is the standard QCD color factor for the product of amplitudes $\mathcal{A}_{i}^{*} \mathcal{A}_{j}$. $C_{I}^{i j}$ and $C_{F_{c}}^{i j}$ are color factors for the diagrams with the initial-state and final-state interactions respectively. Since the IS/FS interactions occur through eikonal soft gluons, they do not affect the kinematics of the relevant diagrams and we can retain the same product of amplitudes $\mathcal{A}_{i}^{*} \mathcal{A}_{j}$, with the new modified color factors, $\left(C_{I}^{i j}+C_{F_{c}}^{i j}\right) / C_{U}^{i j}$, giving the appropriate color flow. Collecting the color factors and the bilinear amplitudes in Eq. (13), we can define the modified partonic hard parts,

$$
H_{q b \rightarrow c d}^{\bmod } \equiv \sum_{i, j} \frac{C_{I}^{i j}+C_{F_{c}}^{i j}}{C_{U}^{i j}} \mathcal{A}_{i}^{*} \mathcal{A}_{j}
$$

While in Ref. [16] only quark initiated subprocesses were considered, in Ref. [19] the CGI-GPM approach was extended to gluon initiated processes. They calculated the effects of the ISIs/FSIs relevant for $p^{\uparrow} p \rightarrow J / \psi+X$ and $p^{\uparrow} p \rightarrow D+X$. For the case of gluons, the substitution required is

$$
\begin{aligned}
f_{1 T}^{\perp g} H_{U}^{g b \rightarrow c d} \equiv & f_{1 T}^{\perp g} \sum_{i, j} \mathcal{A}_{i}^{*} \mathcal{A}_{j} \\
\rightarrow & \sum_{i, j} \frac{C_{I}^{(f) i j}+C_{F_{c}}^{(f) i j}}{C_{U}^{i j}} f_{1 T}^{\perp g(f)} \mathcal{A}_{i}^{*} \mathcal{A}_{j} \\
& +\frac{C_{I}^{(d) i j}+C_{F_{c}}^{(d) i j}}{C_{U}^{i j}} f_{1 T}^{\perp g(d)} \mathcal{A}_{i}^{*} \mathcal{A}_{j} .
\end{aligned}
$$

Here things are different compared to the quark case since in the CGI-GPM framework, the process-dependent gluon Sivers function can be written as a linear combination of two independent universal gluon distributions $f_{1 T}^{\perp g(f)}$ and $f_{1 T}^{\perp g(d)}$. These two gluon Sivers functions correspond to two possible ways of contracting the color indices of the three gluon fields in the operator definition of the gluon Sivers function. The $f$-type denotes a completely antisymmetric contraction, $-i f_{a b c}$, and the $d$-type denotes a completely symmetric contraction, $d_{a b c}$. We therefore define two modified hard parts, one associated with the $f$-type GSF and the other associated with the $d$-type GSF:

$$
H_{g b \rightarrow c d}^{(f / d)} \equiv \sum_{i, j} \frac{C_{I}^{(f / d) i j}+C_{F_{c}}^{(f / d) i j}}{C_{U}^{i j}} \mathcal{A}_{i}^{*} \mathcal{A}_{j} .
$$


The two GSFs $f_{1 T}^{\perp g(f)}$ and $f_{1 T}^{\perp g(d)}$ have different properties. They have different behaviors under charge conjugation. $f_{1 T}^{\perp g(f)}$ is $C$-even and $f_{1 T}^{\perp g(d)}$ is $C$-odd. Therefore only $f_{1 T}^{\perp g(f)}$ is constrained by the Burkardt sum rule, which is defined in terms of $C$-even operators. We will be looking at bounds on the gluon contribution to $A_{N}$, from the BSR, both in the GPM and CGI-GPM frameworks. In this work, we calculate the effects of the initial- and final-state interactions for both direct photon production and photon production via fragmentation from quarks, following the techniques of Ref. [19].

\section{A. Modified hard parts for direct photon production}

In direct photon production, there are no final-state interactions since the partonic final state is a photon, which is colorless. The only partonic subprocesses which give access to the GSF at leading order are $g q \rightarrow \gamma q$ and $g q \rightarrow \gamma \bar{q}$. Here, the first parton to the left of the arrow in the subprocess label (in this case, the gluon) is the one coming from the polarized proton. We have calculated the modified hard parts for these subprocesses in the CGIGPM framework. While the two subprocesses have the same cross section, they receive different modifications in the CGI-GPM framework due to their differing color structures. The unpolarized and the modified hard parts are given below:

$$
\begin{aligned}
& H_{g q \rightarrow \gamma q}^{U}=-\frac{e_{q}^{2}}{N_{c}}\left[\frac{\hat{u}}{\hat{s}}+\frac{\hat{s}}{\hat{u}}\right] \\
& H_{g q \rightarrow \gamma q}^{(f)}=H_{g \bar{q} \rightarrow \gamma \bar{q}}^{(f)}=-\frac{1}{2} H_{g q \rightarrow \gamma q}^{U} \\
& H_{g q \rightarrow \gamma q}^{(d)}=-H_{g \bar{q} \rightarrow \gamma \bar{q}}^{(d)}=\frac{1}{2} H_{g q \rightarrow \gamma q}^{U} .
\end{aligned}
$$

Let us note a few things. Firstly, the modified hard parts are all half the magnitude of the unpolarized hard part. Secondly the hard part associated with the $f$-type GSF has a negative sign with respect to $H^{U}$ for processes with both quarks and antiquarks from the unpolarized proton, whereas the hard part associated with the $d$-type GSF retains the same sign as $H^{U}$ for quarks and has a negative sign for antiquarks.

The quark Sivers functions also contribute to direct photon production via the following subprocesses: $q g \rightarrow$ $\gamma q$ and $q \bar{q} \rightarrow \gamma g$. The relevant hard parts in this case are available in Ref. [16] and we have reproduced them here for the sake of completeness. These are

$$
\begin{aligned}
& H_{q g \rightarrow \gamma q}^{U}=-\frac{e_{q}^{2}}{N_{c}}\left(\frac{\hat{t}}{\hat{s}}+\frac{\hat{s}}{\hat{t}}\right) \\
& H_{q g \rightarrow \gamma q}^{\bmod }=-H_{\bar{q} g \rightarrow \gamma \bar{q}}^{\bmod }=\frac{N_{c}}{N_{c}^{2}-1} e_{q}^{2}\left(\frac{\hat{t}}{\hat{s}}+\frac{\hat{s}}{\hat{t}}\right)
\end{aligned}
$$

$$
\begin{aligned}
& H_{q \bar{q} \rightarrow \gamma g}^{U}=\frac{N_{c}^{2}-1}{N_{c}^{2}} e_{q}^{2}\left(\frac{\hat{u}}{\hat{t}}+\frac{\hat{t}}{\hat{u}}\right) \\
& H_{q \bar{q} \rightarrow \gamma g}^{\bmod }=-H_{\bar{q} q \rightarrow \gamma g}^{\bmod }=\frac{e_{q}^{2}}{N_{c}^{2}}\left(\frac{\hat{u}}{\hat{t}}+\frac{\hat{t}}{\hat{u}}\right) .
\end{aligned}
$$

Note that for the process $q g \rightarrow \gamma q$ the modified hard part is roughly similar in size and has a negative sign for quarks and positive sign for antiquarks (relative to $H^{U}$ ). For the process $q \bar{q} \rightarrow \gamma g$ the modified hard part is much smaller (by a factor of 8 ) and has a positive sign for quarks and a negative sign for antiquarks (relative to $H^{U}$ ). As we will see, this has significant consequences for the SSAs.

\section{B. Modified hard parts for photon production from fragmentation}

For photon production via fragmentation at leading order, there are seven processes that give access to the GSF: $g q \rightarrow q g, g \bar{q} \rightarrow \bar{q} g, g q \rightarrow g q, g \bar{q} \rightarrow g \bar{q}, g g \rightarrow g g$, $g g \rightarrow q \bar{q}$, and $g g \rightarrow \bar{q} q$. The modified hard parts for the first five of these processes are not available in the literature and we have calculated them. We first give the hard parts for $g q \rightarrow q g$ and $g \bar{q} \rightarrow \bar{q} g$ :

$$
\begin{aligned}
& H_{g q \rightarrow q g}^{U}=-\frac{\left(\hat{s}^{2}+\hat{t}^{2}\right)}{2 \hat{s} \hat{t} \hat{u}^{2}}\left[\hat{s}^{2}+\hat{t}^{2}-\frac{\hat{u}^{2}}{N_{c}^{2}}\right] \\
& H_{g q \rightarrow q g}^{(f)}=H_{g \bar{q} \rightarrow \bar{q} g}^{(f)}=-\frac{\left(\hat{s}^{2}+\hat{t}^{2}\right)}{4 \hat{s} \hat{t} \hat{u}^{2}}\left[2 \hat{t} \hat{u}+\hat{u}^{2}\right] \\
& H_{g q \rightarrow q g}^{(d)}=-H_{g \bar{q} \rightarrow \bar{q} g}^{(d)}=-\frac{\left(\hat{s}^{2}+\hat{t}^{2}\right)}{4 \hat{s} \hat{t} \hat{u}^{2}}\left[\hat{s}^{2}+\hat{t}^{2}-2 \frac{\hat{u}^{2}}{N_{c}^{2}}\right] .
\end{aligned}
$$

The hard parts for $g q \rightarrow g q$ and $g \bar{q} \rightarrow g \bar{q}$ are as follows:

$$
\begin{aligned}
& H_{g q \rightarrow g q}^{U}=-\frac{\left(\hat{s}^{2}+\hat{u}^{2}\right)}{2 \hat{s}^{2} \hat{u}}\left[\hat{s}^{2}+\hat{u}^{2}-\frac{\hat{t}^{2}}{N_{c}^{2}}\right] \\
& H_{g q \rightarrow g q}^{(f)}=H_{g \bar{q} \rightarrow g \bar{q}}^{(f)}=-\frac{\left(\hat{s}^{2}+\hat{u}^{2}\right)}{4 \hat{s} \hat{t}^{2} \hat{u}}\left[\hat{s}^{2}+\frac{\hat{t}^{2}}{N_{c}^{2}}\right] \\
& H_{g q \rightarrow g q}^{(d)}=-H_{g \bar{q} \rightarrow g \bar{q}}^{(d)}=+\frac{\left(\hat{s}^{2}+\hat{u}^{2}\right)}{4 \hat{s} \hat{t}^{2} \hat{u}}\left[\hat{s}^{2}-2 \hat{u}^{2}+\frac{\hat{t}^{2}}{N_{c}^{2}}\right] .
\end{aligned}
$$

The hard parts for $g g \rightarrow g g$ are

$$
\begin{aligned}
& H_{g g \rightarrow g g}^{U}=\frac{4 N_{c}^{2}}{N_{c}^{2}-1} \frac{\left(\hat{t}^{2}+\hat{t} \hat{u}+\hat{u}^{2}\right)^{3}}{\hat{s}^{2} \hat{t}^{2} \hat{u}^{2}} \\
& H_{g g \rightarrow g g}^{(f)}=\frac{N_{c}^{2}}{N_{c}^{2}-1} \frac{\left(\hat{t}^{2}+\hat{t} \hat{u}+\hat{u}^{2}\right)^{2}}{\hat{s}^{2} \hat{t}^{2} \hat{u}^{2}}\left(2 \hat{t} \hat{u}+\hat{u}^{2}\right) \\
& H_{g g \rightarrow g g}^{(d)}=0 .
\end{aligned}
$$

Finally, the hard parts for $g g \rightarrow q \bar{q}$ and $g g \rightarrow \bar{q} q$ were calculated in Ref. [19] and are reproduced below for the sake of completeness: 


$$
\begin{aligned}
H_{g g \rightarrow q \bar{q}}^{U}= & \frac{N_{c}}{N_{c}^{2}-1} \frac{1}{\hat{t} \hat{u}}\left(\frac{N_{c}^{2}-1}{2 N_{c}^{2}}-\frac{\hat{t} \hat{u}}{\hat{s}^{2}}\right)\left(\hat{t}^{2}+\hat{u}^{2}\right) \\
H_{g g \rightarrow q \bar{q}}^{(f)}= & H_{g g \rightarrow \bar{q} q}^{(f)}=-\frac{N_{c}}{4\left(N_{c}^{2}-1\right)} \frac{1}{\hat{t} \hat{u}}\left(\frac{\hat{t}^{2}}{\hat{s}^{2}}+\frac{1}{N_{c}^{2}}\right)\left(\hat{t}^{2}+\hat{u}^{2}\right) \\
H_{g g \rightarrow q \bar{q}}^{(d)}= & -H_{g g \rightarrow \bar{q} q}^{(d)}=-\frac{N_{c}}{4\left(N_{c}^{2}-1\right)} \frac{1}{\hat{t} \hat{u}}\left(\frac{\hat{t}^{2}-2 \hat{u}^{2}}{\hat{s}^{2}}+\frac{1}{N_{c}^{2}}\right) \\
& \times\left(\hat{t}^{2}+\hat{u}^{2}\right) .
\end{aligned}
$$

The relevant hard parts for quark initiated subprocesses that give access to the QSFs can be found in Ref. [16]. We do not present them here.

\section{PARAMETRIZATION OF THE TMDS}

In this section we give the details of the functional forms and parameters that we use for the TMDs. For the unpolarized TMDs we adopt the commonly used form with the collinear PDF multiplied by a Gaussian transversemomentum dependence,

$$
f_{i / p}\left(x, k_{\perp} ; Q\right)=f_{i / p}(x, Q) \frac{1}{\pi\left\langle k_{\perp}^{2}\right\rangle} e^{-k_{\perp}^{2} /\left\langle k_{\perp}^{2}\right\rangle}
$$

with $\left\langle k_{\perp}^{2}\right\rangle=0.25 \mathrm{GeV}^{2}$. As with the unpolarized densities, we use a similar factorized Gaussian form for the photon fragmentation function,

$$
D_{\gamma / c}\left(z, \boldsymbol{k}_{\gamma}\right)=D_{\gamma / c}(z) \frac{1}{\pi\left\langle k_{\perp \gamma}^{2}\right\rangle} e^{-k_{\gamma}^{2} /\left\langle k_{\perp \gamma}^{2}\right\rangle}
$$

with $\left\langle k_{\perp \gamma}^{2}\right\rangle=0.25 \mathrm{GeV}^{2}$.

Since we give predictions using the GSF fits of Ref. [22], we adopt the functional form of the Sivers functions used therein:

$$
\begin{aligned}
\Delta^{N} f_{i / p^{\uparrow}}\left(x, k_{\perp} ; Q\right)= & 2 \mathcal{N}_{i}(x) f_{i / p}(x, Q) \frac{\sqrt{2 e}}{\pi} \\
& \times \sqrt{\frac{1-\rho}{\rho}} k_{\perp} \frac{e^{-k_{\perp}^{2} / \rho\left\langle k_{\perp}^{2}\right\rangle}}{\left\langle k_{\perp}^{2}\right\rangle^{3 / 2}}
\end{aligned}
$$

with $0<\rho<1$. Here $\mathcal{N}_{i}(x)$ parametrizes the $x$-dependence of the Sivers function and is generally written as

$$
\mathcal{N}_{i}(x)=N_{g} x^{\alpha_{i}}(1-x)^{\beta_{i}} \frac{\left(\alpha_{i}+\beta_{i}\right)^{\alpha_{i}+\beta_{i}}}{\alpha_{i}^{\alpha_{i}} \beta_{i}^{\beta_{i}}} .
$$

For the Sivers function to satisfy the positivity bound,

$$
\frac{\left|\Delta^{N} f_{i / p^{\uparrow}}\left(x, \mathbf{k}_{\perp}\right)\right|}{2 f_{i / p}\left(x, \mathbf{k}_{\perp}\right)} \leq 1 \quad \forall x, \mathbf{k}_{\perp},
$$

it is necessary to have $\left|\mathcal{N}_{i}(x)\right|<1$.
In order to study the efficacy of the probe, we explore the following choices for the Sivers functions:

(1) Quark and gluon Sivers functions with the positivity bound saturated, viz. $\mathcal{N}_{i}(x)=1$ and $\rho=2 / 3$.

(2) The SIDIS1 [37] and SIDIS2 [38] fits of the QSFs, along with the associated GSF fits from Ref. [22].

The first choice, which we will refer to as the "saturated" Sivers function, is the maximal Sivers function allowed by the positivity bound for a fixed width $\left\langle k_{\perp}^{2}\right\rangle$ and $\rho$, with a particular choice of unpolarized collinear gluon density. The parameter $\rho$ is set to $2 / 3$ in order to maximize the first $k_{\perp}$-moment of the Sivers function, following Ref. [43]. Using the saturated Sivers functions for quarks and gluons allows us to study the general kinematic dependencies of the asymmetry and the relative importances of the quark and gluon contributions to the asymmetry. It also lets us study how uncertainties in the knowledge of the collinear, unpolarized gluon and sea quark densities might impact the probe.

SIDIS1 [37] and SIDIS2 [38] are two different sets of QSFs both fitted to data on single-spin asymmetry in SIDIS. The SIDIS1 set was fit to data on pion production at HERMES and flavor unsegregated data on positive hadron production at COMPASS. They used quark fragmentation functions by Kretzer [44]. The data, being flavor unsegregated, were not sensitive to sea quark contributions. Hence this set contains parametrizations for only $u$ and $d$ quark Sivers functions. The SIDIS2 set was fit to flavorsegregated data on hadron production at HERMES and COMPASS. Since strange meson production receives a contribution from sea quarks, this fit includes sea quark Sivers functions as well. Further they used fragmentation functions by de Florian, Sassot and Stratmann (DSS) [45] in this second fit.

Associated with these two QSF sets are the two fits of the GSF from Ref. [22], which we will refer to as SIDIS1 and SIDIS2 as well. These were both obtained by constraining the gluon contribution to data on $A_{N}$ in midrapidity pion production at the RHIC [46], with the aforementioned QSFs being used to account for the quark contribution. While both the GSFs - along with their associated QSF sets-give good fits to the data on midrapidity pion production, they have very different $x$-dependencies. SIDIS1 is larger in the moderate- $x$ region and SIDIS2 is larger in the low- $x$ region. The values of the parameters of the two QSF sets as well as the associated GSF fits are given in Table I.

\section{NUMERICAL ESTIMATES FOR DIRECT PHOTON PRODUCTION}

In this work we wish to see if prompt photon production at RHIC can be used to obtain any information on the gluon Sivers function and whether it has any discriminating power between the available GSF parametrizations. To this end we need to consider various things such as the 
TABLE I. Parameters for the various Sivers function fits used.

\begin{tabular}{lcl}
\hline \hline & SIDIS2 QSFs from Ref. [38] & \\
\hline$N_{u}=0.35$ & $N_{d}=-0.90$ & $N_{s}=-0.24$ \\
$N_{\bar{u}}=0.04$ & $N_{\bar{d}}=-0.40$ & $N_{\bar{s}}=1$ \\
$\alpha_{u}=0.73$ & $\alpha_{d}=1.08$ & $\alpha_{\text {sea }}=0.79$ \\
$\beta=3.46$ & $M_{1}^{2}=0.34 \mathrm{GeV}^{2}$ & \\
\hline \hline
\end{tabular}

\begin{tabular}{ll}
\hline \hline & SIDIS1 QSFs from Ref. [37] \\
\hline$N_{u}=0.32$ & $N_{d}=-1.00$ \\
$\alpha_{u}=0.73$ & $\alpha_{d}=1.08$ \\
$\beta_{u}=0.53$ & $\beta_{d}=3.77$ \\
\hline \hline
\end{tabular}

SIDIS2 GSF from Ref. [22]

\begin{tabular}{llll}
\hline$N_{g}=0.05$ & $\alpha_{g}=0.8$ & $\beta_{g}=1.4$ & $\rho=0.576$ \\
\hline \hline
\end{tabular}

SIDIS1 GSF from Ref. [22]

\begin{tabular}{llll}
\hline$N_{g}=0.65$ & $\alpha_{g}=2.8$ & $\beta_{g}=2.8$ & $\rho=0.687$ \\
\hline \hline
\end{tabular}

kinematics; contribution of QSFs to the asymmetry; uncertainties in the knowledge of the collinear, unpolarized PDFs; existing bound on the GSFs; etc.

Direct photon production at $x_{F}<0$ was analyzed in Ref. [35]. The authors noted that this region is dominated by the Compton scattering process $g q \rightarrow \gamma q$ with the gluons coming from the transversely polarized proton. Hence $x_{F}<0$ had been identified as the region appropriate for probing the GSF. They had then suggested, in general, to use the large- $P_{T}$ region in the backward hemisphere. In Fig. 3, we show the unpolarized Lorentz-invariant cross section for the production of prompt photons at RHIC energy, $\sqrt{s}=200 \mathrm{GeV}$, as a function of $x_{F}$, at fixed $P_{T}=$ $5 \mathrm{GeV}$ (left panel) and as a function of $P_{T}$ at a fixed pseudorapidity $\eta=-2$. By prompt photons, we mean both direct and fragmentation photons. The plot also shows the direct photon contribution separately. The inclusive components are given as thin lines and the direct components are given as thick lines. For both the inclusive and direct components, the contribution from subprocesses with the gluon in the transversely polarized proton is indicated separately. Further for the direct component, the contributions from subprocesses with quarks, as well as sea quarks in the transversely polarized proton, are indicated separately. In obtaining these numbers, we used CTEQ6L [47] PDFs for the collinear part of the proton densities and the BFGII [48] parton-to-photon FFs for the collinear part of the fragmentation functions. A Gaussian width $\left\langle k_{\perp}^{2}\right\rangle=$ $0.25 \mathrm{GeV}^{2}$ was used for both gluon and quark TMD-PDFs as well as for the TMD photon FFs. The renormalization and factorization scales were chosen to be $Q=P_{T}$.

As we can see from Fig. 3, the total prompt photon cross section inclusive of direct and fragmentation photons is of the same order of magnitude as the direct photon cross section alone. Overall, gluons dominate the production process in the kinematic regions we consider. Of the direct photon component, for $P_{T} \gtrsim 3 \mathrm{GeV}$ and $x_{F} \lesssim-0.1$, more than $75 \%$ of the cross section is from the Compton process $g q \rightarrow \gamma q$ with the initial-state gluon from the forward-going proton. Among the contributions to direct photons coming from quark-initiated processes (processes with quarks in the transversely polarized proton), the sea quarks give the dominant component, being around $10 \%-15 \%$ of the total
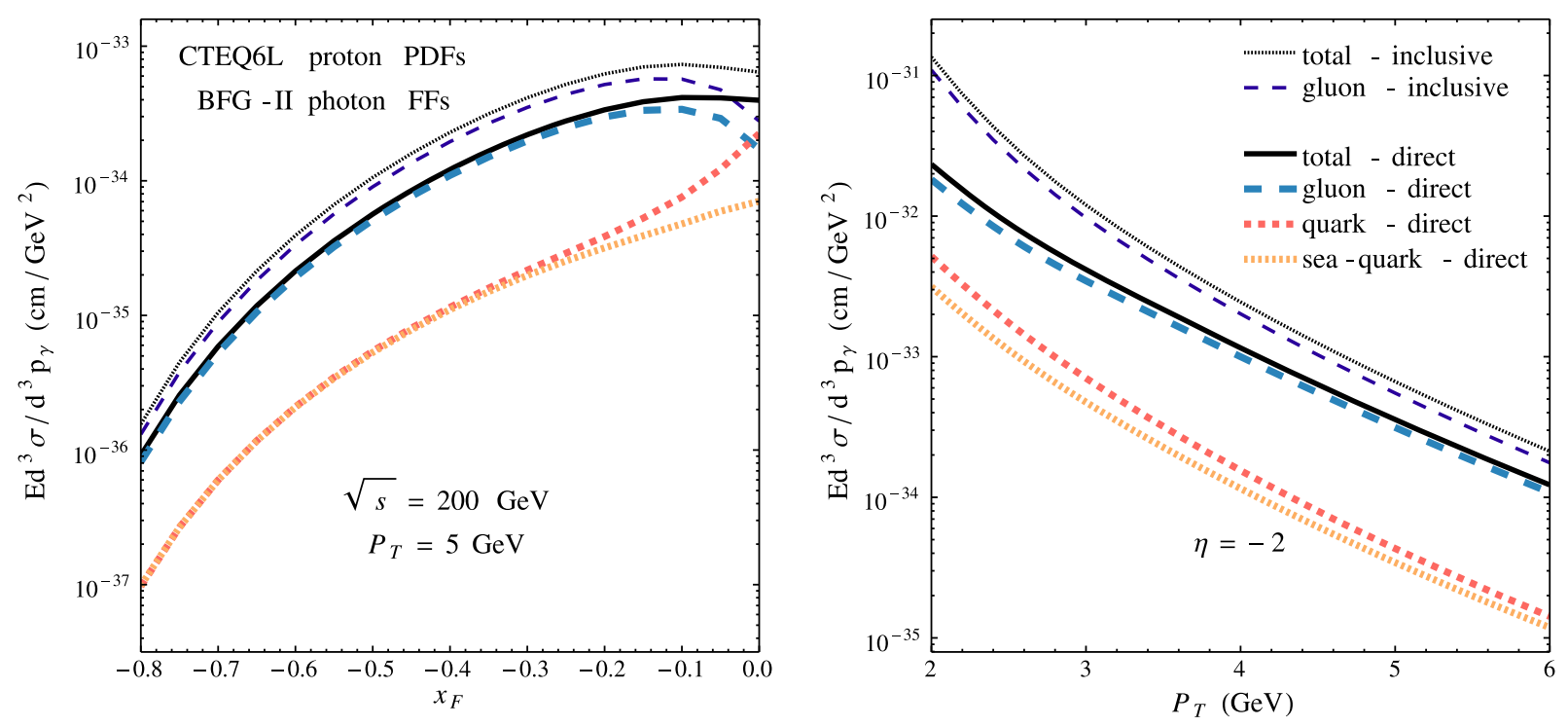

FIG. 3. Unpolarized Lorentz-invariant cross section for prompt photon production at the RHIC $(\sqrt{s}=200 \mathrm{GeV})$ as a function of $x_{F}$ (at $P_{T}=5 \mathrm{GeV}$, left panel) and $P_{T}$ (at rapidity $\eta=-2$, right panel). The thick lines indicate the direct photon contributions and the thin lines indicate the inclusive (direct and fragmentation) prompt photon contributions. The renormalization and factorization scales were chosen to be $Q=P_{T}$. 
cross section. In the low $P_{T}(\lesssim 3 \mathrm{GeV})$ and $x_{F}>-0.1$ regions, where the cross section is highest, the contributions from gluons, valence quarks and sea quarks are similar in magnitude; hence precise knowledge of the collinear densities will be required for any analysis of the SSA in this kinematic region. The qualitative details of the above observations remain unchanged even when we use other available fits of the collinear densities.

\section{A. GPM}

\section{Asymmetry estimates using saturated Sivers functions}

We now consider results for the asymmetry obtained using saturated Sivers functions for gluons and quarks. As mentioned in Sec. IV, the saturated Sivers function can be taken to be an upper bound on the Sivers function as allowed by the positivity bound, Eq. (28). Considering the asymmetries obtained using the saturated Sivers functions is useful for two reasons. First it allows us to see the maximum possible sizes of the effect and consider the relative importances of the valence quarks, sea quarks and gluons in the absence of any inputs from fits of the QSFs and the GSF. Second, by considering different choices of collinear densities we can get an idea of how uncertainties in the knowledge of the collinear PDFs can affect the analysis. We will consider both now.

In Fig. 4, we show the asymmetries obtained in the GPM framework using saturated Sivers functions for the gluon and all flavors of quarks. A positive sign is used for QSFs of all flavors. We show the asymmetry in the direct photons alone, as well as the asymmetry inclusive of direct and fragmentation photons. The inclusive photon asymmetries are given as thin lines and the direct photon asymmetries are given as thick lines. The gluon and quark contributions for both are shown separately. In the bottom panels, we show the percentage change in direct photon results when using different two different choices of LO collinear densities, MRST2001LO [49] and GRV98LO [50].
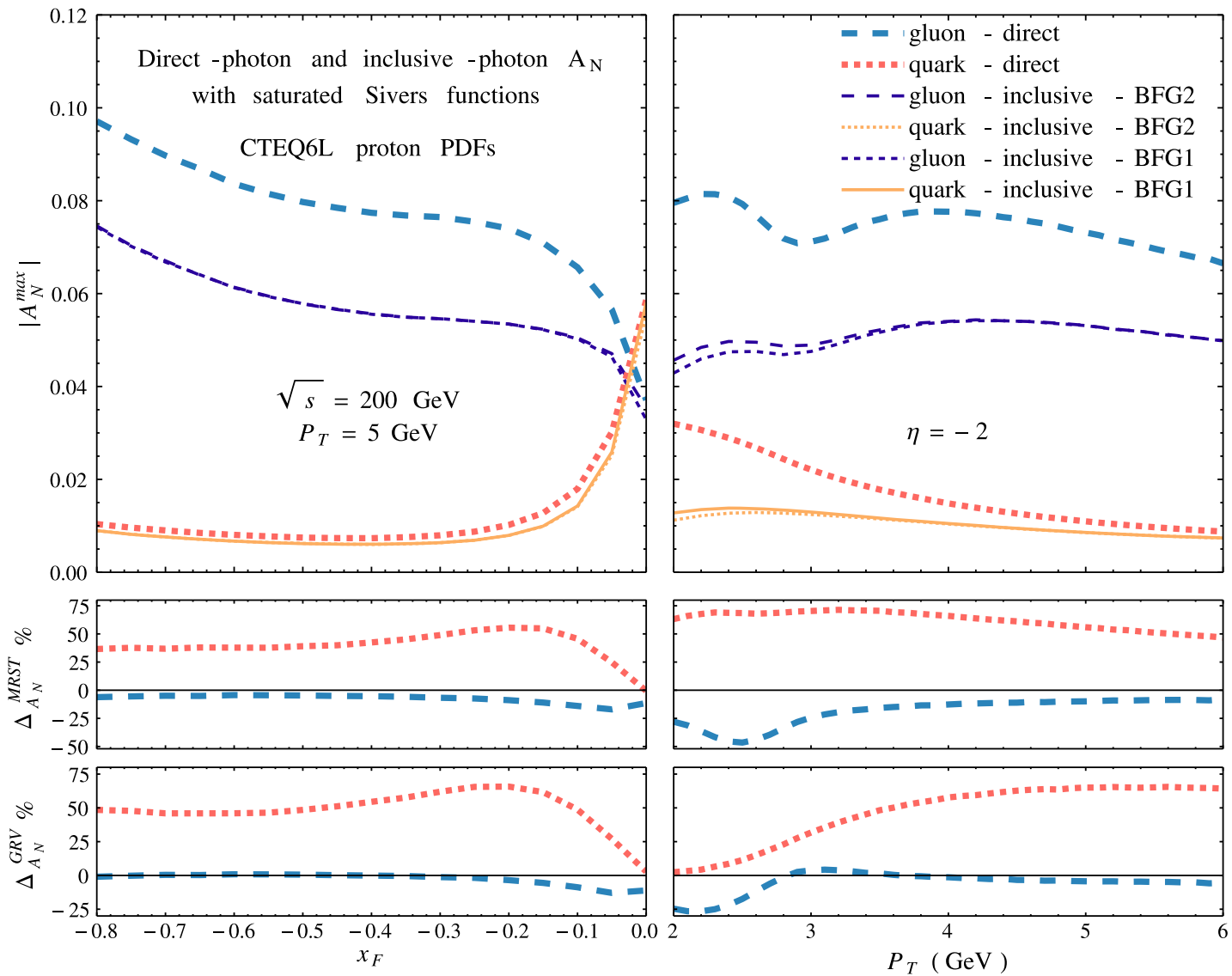

FIG. 4. SSA in prompt photon production using saturated quark and gluon Sivers functions. Results shown as a function of $x_{F}$ (at $P_{T}=5 \mathrm{GeV}$, left panel) and $P_{T}$ (at rapidity $\eta=-2$, right panel). Thick lines indicate asymmetry in direct photons and thin lines indicate asymmetry in all prompt photons inclusive of fragmentation photons. The top pair of panels shows the results obtained using CTEQ6L [47] PDFs. The pair of panels below them indicates the percentage change in the direct photon results when MRST2001LO [49] PDFs are used, i.e., $100 \times\left(A_{N}^{\mathrm{CTEQ}}-A_{N}^{\mathrm{MRST}}\right) / A_{N}^{\mathrm{MRST}}$. The pair of panels further below shows the percentage change in direct photon results when GRV98LO [50] PDFs are used. 
We first discuss the direct photon results. When using CTEQ6L PDFs, the saturated GSF (with QSFs set to zero) gives an asymmetry of up to almost $10 \%$ at $x_{F}=-0.8$ and $8 \%$ at $P_{T}=2 \mathrm{GeV}$. The asymmetry from the saturated QSFs is largest at low values of $\left|x_{F}\right|$ with $P_{T}$ being $6 \%$ at $x_{F}=0$ and $3 \%$ at $P_{T}=2 \mathrm{GeV}$. It can be seen from the plot of the cross section in Fig. 3 that the contribution of sea quarks is significant in the entire kinematics range and is in fact dominant for $x_{F}<-0.2$ at $P_{T}=5 \mathrm{GeV}$.

From the bottom two sets of panels of Fig. 4, we can see how the direct photon results vary when using the MRST2001LO and GRV98LO PDF sets for the collinear parts of the TMDs. This gives us an idea of how uncertainties in our knowledge of the collinear densities can impact the predictions of SSA in the considered kinematic region. Both MRST and GRV give quark contributions that are larger by $50 \%-70 \%$, whereas gluon contributions are mostly similar throughout the kinematic range except at low- $P_{T}$ values at $\eta=2$ where MRST gives a gluon contribution that is smaller by up to $50 \%$ and GRV gives a gluon contribution that is smaller by up to $25 \%$.

Finally we consider the fragmentation contributions. Since it is important to get an idea of the maximum impact that fragmentation photons can have on the asymmetry, we look at the asymmetry inclusive of both direct and fragmentation photons. We have plotted the GSF-only and QSF-only contributions of inclusive asymmetry for two different choices of parton-to-photon FFs, BFGI and BFGII. As can be seen from the thin violet curves in Fig. 4, the GSF contribution of the inclusive asymmetry is diluted by anywhere between $10 \%$ and $50 \%$ of the direct photon value. The QSF contribution to the inclusive asymmetry also has the same fate. Hence, it is important to remove the fragmentation contribution as much as possible. Overall the results for the inclusive asymmetry do not depend much on the choice of the FF set used. Results obtained for the inclusive asymmetry for different choices of collinear PDFs also show a similar trend.

\section{Asymmetry estimates using existing fits as well as constraints from the Burkardt sum rule}

We now consider existing information on the gluon and quark Sivers functions that have been obtained from fits to data. As mentioned in Sec. IV, we consider two different sets of the QSFs, labeled SIDIS2 and SIDIS1, along with their associated GSF fits from Ref. [22]. Apart from the two available fits of the GSF, a general indirect bound on the GSF can be obtained based on the Burkardt sum rule [39]. This sum rule for the Sivers functions is essentially the requirement that the net transverse momentum of all the partons in a transversely polarized proton must vanish,

$$
\left\langle\boldsymbol{k}_{\perp}\right\rangle=\sum_{i=q, \bar{q}, g}\left\langle\boldsymbol{k}_{\perp i}\right\rangle=\sum_{i=q, \bar{q}, g} \int d x f_{1 T}^{\perp(1) i}(x)=0
$$

where $f_{1 T}^{\perp(1) i}$ is the first transverse moment of the Sivers function,

$$
f_{1 T}^{\perp(1) i} \equiv-\int d^{2} \boldsymbol{k}_{\perp} \frac{k_{\perp}}{4 M_{p}} \Delta^{N} f_{g / p^{\uparrow}}\left(x, k_{\perp}\right) .
$$

The QSF fits of the SIDIS2 set, taken with their associated errors, allow the gluon contribution to be in the following range:

$$
-10 \leq\left\langle k_{\perp g}\right\rangle \leq 48 \mathrm{MeV} .
$$

This is to be compared with the following values obtained for the quarks in the SIDIS2 fit:

$$
\left\langle k_{\perp u}\right\rangle=98_{-28}^{+60} \mathrm{MeV}, \quad\left\langle k_{\perp d}\right\rangle=-113_{-51}^{+45} \mathrm{MeV} .
$$

Since this is an indirect constraint based on a quantity integrated over the parton light-cone momentum fraction $x$, this tells us nothing about the $x$-dependence-or for that matter the $k_{\perp}$-dependence-of the GSF. However, we can still get an idea of the possible sizes of the gluon contribution to the asymmetry in direct photon production by considering various possible GSF parameter sets, $N_{g}$, $\alpha_{g}, \beta_{g}$ and $\rho$, that result in a Sivers function obeying Eq. (31). We obtained these sets by performing a scan over the parameter space, varying the parameters in the following ranges: $N_{g}$ in the range -1 to 1 in steps of $0.1, \alpha_{g}$ and $\beta_{g}$ in the range 0 to 4 in steps of 0.2 , and $\rho$ in the range 0.1 to 0.9 in steps of 0.05 . This exercise is justified given the very different $x$-dependencies of the SIDIS1 and SIDIS2 fits of the GSF, both of which were obtained using QSF sets that describe SIDIS data equally well.

In Fig. 5 we plot the band of direct photon $A_{N}$ values obtained with the GSF parameter set results from the scan. This is shown by the light blue shaded region. Along with it, we also plot the quark and gluon contribution to the direct photon asymmetry as given by the SIDIS2 and SIDIS1 sets. For the collinear parts of the densities, we used GRV98LO PDFs as they were used in the extraction of the SIDIS2 fits and the BSR bound. Both the GSF fits give asymmetries that lie well within the asymmetry band given by the constraint in Eq. (31). The indirect bound allows a gluon contribution to the asymmetry of up to $0.07 \%-0.1 \%$. Overall we find that both the SIDIS1 and SIDIS2 GSF fits, as well as the Burkardt sum rule constraints, predict negligible values for the gluon contribution to the asymmetry.

\section{B. CGI-GPM}

\section{Asymmetry estimates using saturated Sivers functions}

We now consider the probe in the context of the colorgauge invariant generalized parton model. 

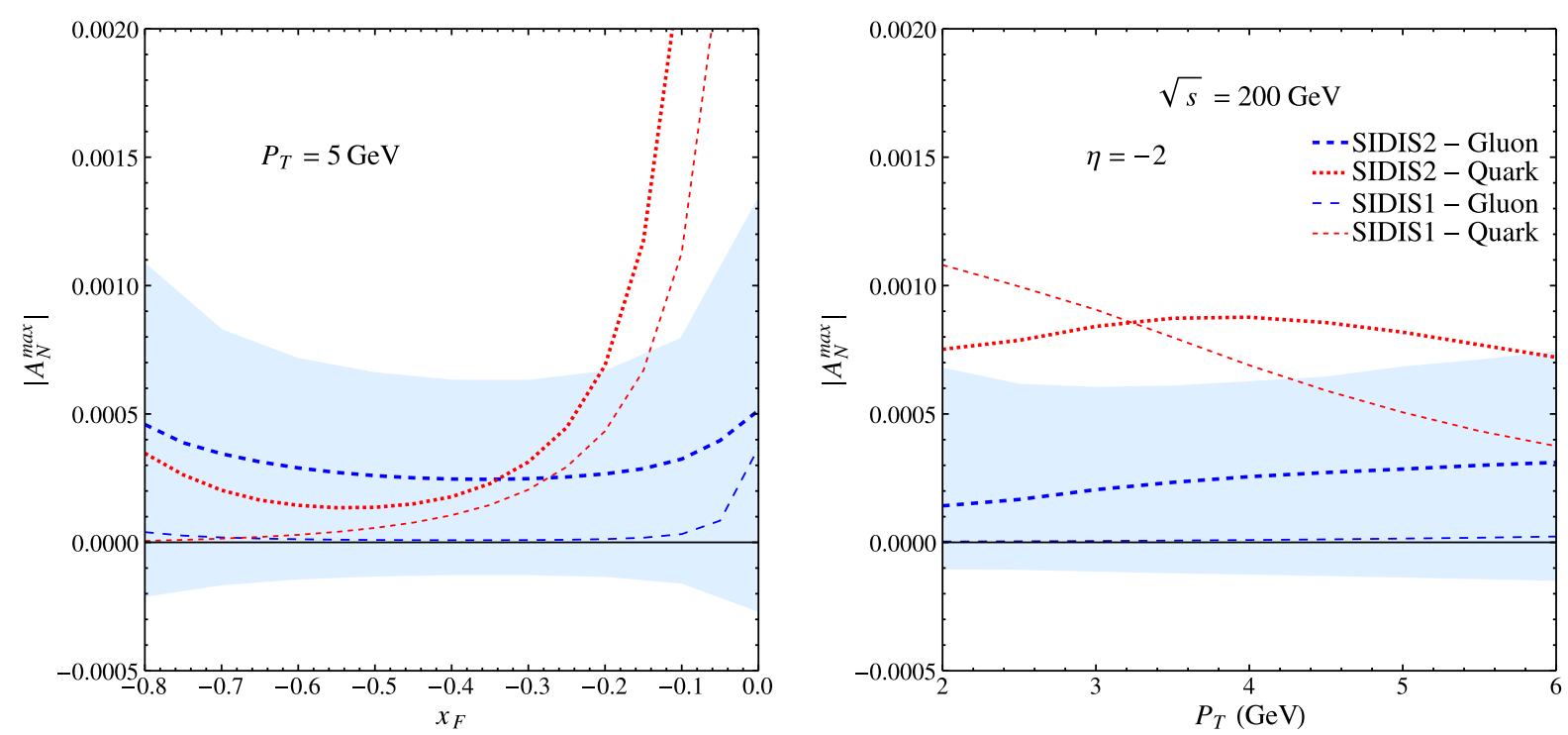

FIG. 5. Burkardt sum rule based constraints on $A_{N}$ in the GPM framework and predictions of asymmetry using the SIDIS2 [38] and SIDIS1 [37] QSFs and associated GSFs [22]. The light blue band shows the envelope of asymmetries obtained from GSFs that obey the BSR based constraint, Eq. (23).

In Fig. 6, we show the asymmetries obtained using saturated Sivers functions, in the CGI-GPM framework. We show the asymmetry in the direct photons alone, as well as the asymmetry inclusive of direct and fragmentation photons.

We first discuss the direct photon results. We can see that the $f$-type contribution has a negative sign. This is because the hard parts $H_{g q(\bar{q}) \rightarrow \gamma q(\bar{q})}^{(f)}$ have an opposite sign with respect to $H_{g q \rightarrow \gamma q}^{U}$, whereas the hard parts $H_{g q(\bar{q}) \rightarrow \gamma q(\bar{q})}^{(d)}$ have the same sign as $H_{g q \rightarrow \gamma q}^{U}$. Since the modified hard parts associated with the two GSFs are half the magnitude of the unpolarized hard part, the asymmetry estimates will be halved in magnitude as compared to the GPM result. Further the $d$-type hard parts have opposite signs for quarks and antiquarks, i.e., $H_{g q \rightarrow \gamma q}^{(d)}=-H_{g \bar{q} \rightarrow \gamma \bar{q}}^{(d)}$, so in regions
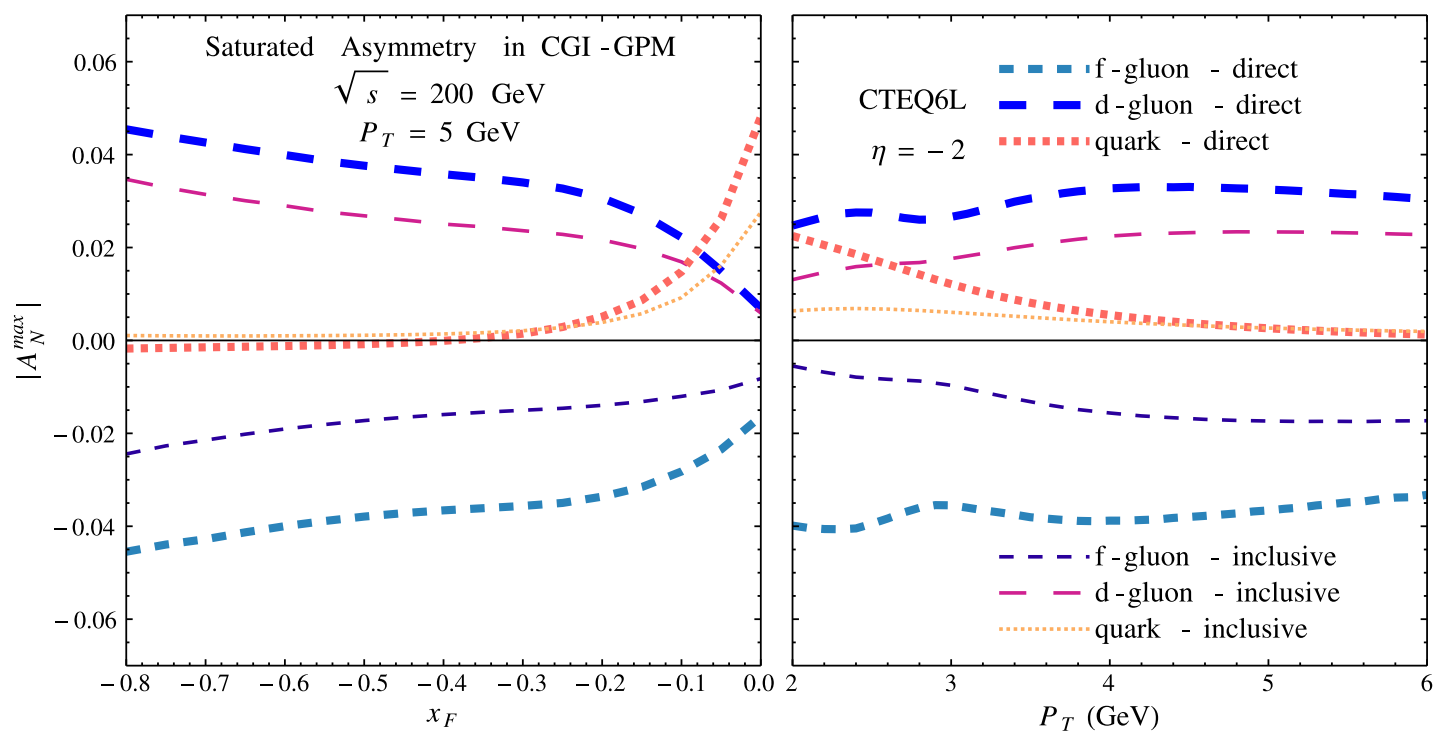

FIG. 6. SSA in direct photon production using the CGI-GPM framework with saturated quark and gluon Sivers functions. The results are shown as a function of $x_{F}$ (at $P_{T}=5 \mathrm{GeV}$, left panel) and $P_{T}$ (at rapidity $\eta=-2$, right panel). Note that direct photon asymmetries from the $f$ and $d$ type GSFs are shown with opposite signs. This is because their modified hard parts associated with them have opposite signs. Depending on the relative signs of the two GSFs and also their relative magnitudes, the contributions from the two GSFs may add up or cancel out. The results were obtained using CTEQ6L PDFs. 
where the antiquark content of the unpolarized proton is significant, there is a further suppression for the $d$-type contribution. This can be clearly seen from the panel for $\eta=-2$. Overall, depending on the relative signs of the two GSFs and also their relative magnitudes, the contributions from the two of them may add up or cancel out.

Unlike the gluon contribution which decreases by a factor of 2 with respect to the GPM, the changes in the quark contribution are not so straightforward. We should first note that in obtaining the above plot, the signs of the saturated $u, d$ and $s$ quark Sivers functions were chosen to be negative, i.e., $\mathcal{N}_{u}(x)=\mathcal{N}_{d}(x)=\mathcal{N}_{s}(x)=-1$. The modified hard part for the process $q g \rightarrow \gamma q$ has a negative sign with respect to the unpolarized hard part; therefore the negative sign ensures that the resulting quark contribution to the asymmetry is positive in regions where $q g \rightarrow \gamma q$ dominates over $q \bar{q} \rightarrow \gamma g$. This is the case in the neighborhood of midrapidity, $x_{F}>-0.2$. For further backward regions, the process $q \bar{q} \rightarrow \gamma g$ is dominant and the quark asymmetries are highly suppressed since the modified hard part for this process is suppressed by a factor of 8 with respect to the unpolarized hard part [cf. Eq. (15)].

Overall for the case of direct photons, the saturated Sivers function based analysis in the CGI-GPM framework leads us to the following conclusions on how things are different with respect to the GPM: both the $f$ and $d$ type GSF contributions to the asymmetry are about half the magnitude of the GPM result. If the two GSFs are similar in magnitude, their overall contributions may add up or cancel each other out depending on their signs, which are unknown. The changes in the quark contributions depend on the various signs involved. In highly backward regions, the quark contribution to the asymmetry is highly suppressed with respect to the gluon contribution. This is because (a) the quark contribution happens through the $q \bar{q} \rightarrow \gamma g$ subprocess which has a much smaller cross section as compared to $q g \rightarrow \gamma q$, and (b) the initial-state interactions contribute a further suppression by a factor of 8 with respect to the standard partonic cross section.

As was the case with the GPM estimates, the results for the inclusive photon asymmetry are somewhat lower compared to the direct photon asymmetry.

\section{Asymmetry estimates using existing fits as well as constraints from the Burkardt sum rule}

In Fig. 7 we plot the range of values for the GSF contribution to the asymmetry in the CGI-GPM framework, as allowed by the constraint from the Burkardt sum rule. We also show the quark contribution to the asymmetry as given by the SIDIS2 QSFs. Since there are no available fits of the GSF in the CGI-GPM framework, we do not show any gluon contribution, except for the asymmetry band allowed by the BSR constraint. It is important to note that the band shown in the plot corresponds only to the contribution of the $f$-type GSF. As mentioned in Sec. III, the BSR does not constrain the $d$-type Sivers function as it is odd under charge conjugation. As was the case with the GPM framework, we find that both the fits as well as the constraints based on the Burkardt sum rule predict negligible values for the gluon contribution to the asymmetry.

Overall, in the CGI-GPM framework we see that asymmetry predictions decrease in magnitude but the relative importance of the gluon contribution increases in the highly backward regions, i.e., $x_{F}<-0.3$.
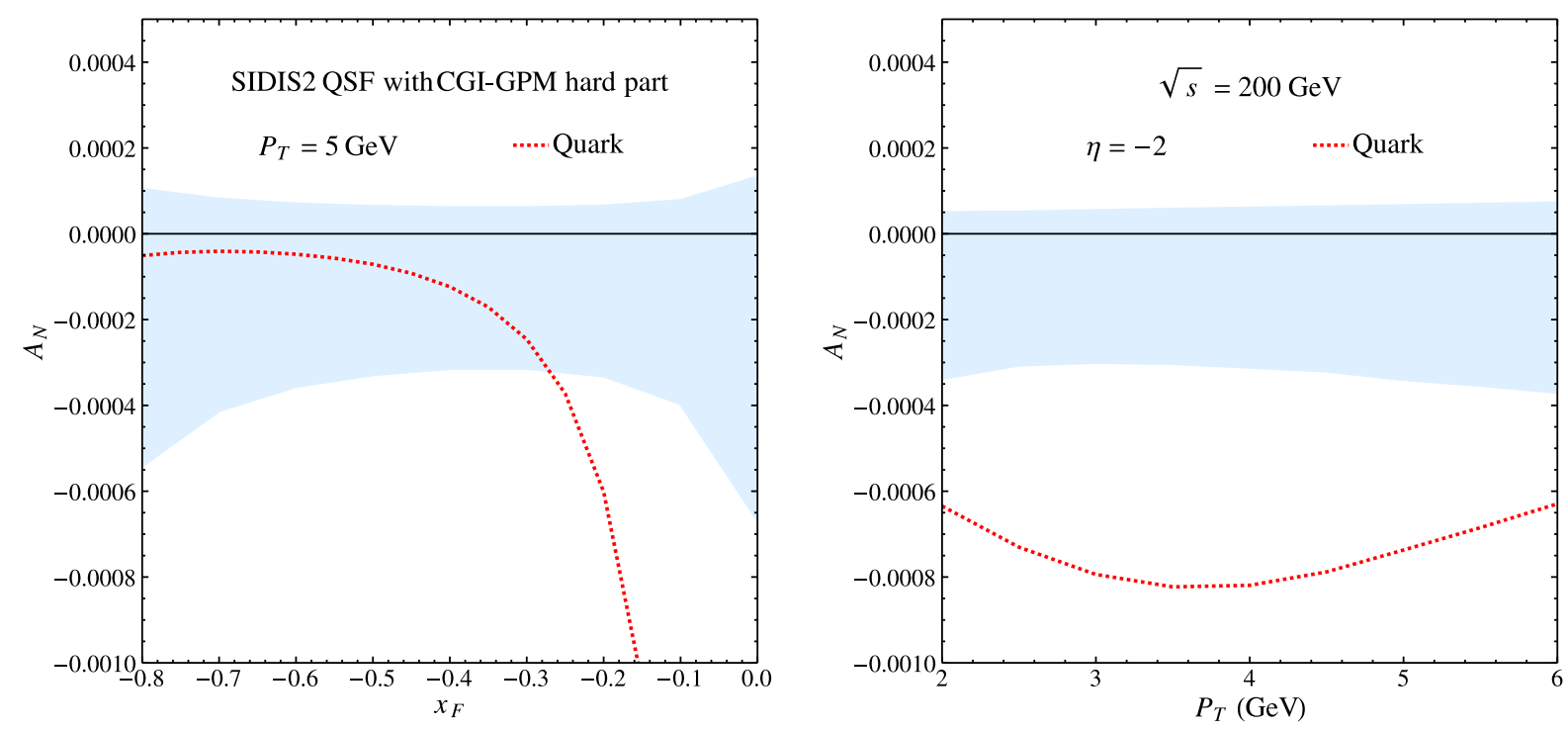

FIG. 7. Burkardt sum rule based constraints on $A_{N}$ in the CGI-GPM framework and predictions of asymmetry using the SIDIS2 [38] QSFs. The light blue band shows the envelope of asymmetries obtained from GSFs that obey the BSR based constraint, Eq. (23). 


\section{CONCLUSIONS}

In this work we have presented results for SSA in the production of prompt photons in the negative rapidity region at the RHIC. In this region, the production of direct photons is dominated by gluons in the transversely polarized proton through the QCD Compton process, $g q \rightarrow \gamma q$. Thus any observed asymmetry in this region could be a strong indication of a nonzero gluon Sivers function. We find that the use of a gluon Sivers function that saturates the positivity bound can lead to an asymmetry in direct photons of up to $10 \%$. We find that inclusion of fragmentation photons can dilute the asymmetries by anywhere between $10 \%$ and $50 \%$ of the value for just the direct photons. A stricter constraint on the GSF than the positivity bound can be obtained using the Burkardt sum rule. In Ref. [38], wherein the SIDIS2 QSFs were extracted, it was found that the BSR allowed an average gluon transverse momentum in the range $-10 \leq\left\langle k_{\perp g}\right\rangle \leq 48 \mathrm{MeV}$. We find that the GSF parametrizations that satisfy this constraint give negligible asymmetries.

Further we also studied the asymmetry in the context of the color-gauge invariant generalized parton model, in which the nonuniversality of the Sivers functions is accounted for by taking into account the effects of the process-dependent initial-state and final-state interactions. We find that both the $f$-type and $d$-type GSFs can contribute to a SSA in direct photon production. Both of them, when saturated, lead to peak direct photon asymmetries of around 5\%. Constraints based on the BSR apply only to the $f$-type GSF and give negligible values for the asymmetry, as was the case with the GPM framework. However the $d$-type GSF is so far not constrained by anything except the positivity bound and hence, on principle, can be much larger.

\section{ACKNOWLEDGMENTS}

The work of R. M. G. is supported by the Department of Science and Technology, India under Grant No. SR/S2/ JCB-64/2007 under the J.C. Bose Fellowship scheme. A. M. would like to thank the Department of Science and Technology, India, for financial support under Grant No. EMR/2014/0000486. R. M. G. wishes to acknowledge the support of the Institute of Physics and Astronomy, Amsterdam for the IPA Visiting Professorship in 2018 and the hospitality of the theory group at NIKHEF, Amsterdam.

\section{APPENDIX: TREATMENT OF GPM KINEMATICS}

In this work, we have considered the single-inclusive production of both direct photons and photons produced via fragmentation of partons. Here we outline the treatment of parton kinematics for both cases.

The momenta of the polarized proton (A), unpolarized proton (B) and the photon can be written in the $p p$ center of mass frame as

$$
\begin{aligned}
& p_{A}=\frac{\sqrt{s}}{2}(1,0,0,1), \quad p_{B}=\frac{\sqrt{s}}{2}(1,0,0,-1) \quad \text { and } \\
& p_{\gamma}=\left(E_{\gamma}=\sqrt{P_{T}^{2}+P_{L}^{2}}, P_{T}, 0, P_{L}\right) .
\end{aligned}
$$

The parton from the polarized proton (a) and the parton from the unpolarized proton (b) carry light-cone momentum fractions $x_{a}=p_{a}^{+} / p_{A}^{+}$and $x_{b}=p_{b}^{-} / p_{B}^{-}$and transverse momenta $\boldsymbol{k}_{\perp a}$ and $\boldsymbol{k}_{\perp b}$ respectively. Taking them both to be on shell, their momenta are given by

$$
\begin{aligned}
p_{a}= & x_{a} \frac{\sqrt{s}}{2}\left(1+\frac{k_{\perp a}^{2}}{x_{a}^{2} s}, \frac{2 k_{\perp a}}{x_{a} \sqrt{s}} \cos \phi_{\perp a},\right. \\
& \left.\frac{2 k_{\perp a}}{x_{a} \sqrt{s}} \sin \phi_{\perp a}, 1-\frac{k_{\perp a}^{2}}{x_{a}^{2} s}\right) \\
p_{b}= & x_{b} \frac{\sqrt{s}}{2}\left(1+\frac{k_{\perp b}^{2}}{x_{b}^{2} s}, \frac{2 k_{\perp b}}{x_{b} \sqrt{s}} \cos \phi_{\perp b},\right. \\
& \left.\frac{2 k_{\perp b}}{x_{b} \sqrt{s}} \sin \phi_{\perp b},-1+\frac{k_{\perp b}^{2}}{x_{b}^{2} s}\right)
\end{aligned}
$$

where $\phi_{\perp a}$ and $\phi_{\perp b}$ are the azimuthal angles of the parton transverse momenta.

In the case of direct photon production, the treatment of parton kinematics is relatively simple as the on-shell condition $\hat{s}+\hat{t}+\hat{u}=0$ can be used to fix one of the variables, such as $x_{a}$ or $x_{b}$. In addition, we have the requirement that the energy of the incoming parton should not be greater than that of its parent particle, $E_{a(b)} \leq E_{A(B)}$. This leads to the following constraint,

$$
k_{\perp a(b)}<\sqrt{s} \min \left[x_{a(b)}, \sqrt{x_{a(b)}\left(1-x_{a(b)}\right)}\right] .
$$

In the case of photon production via fragmentation, the transverse momentum in the fragmentation makes the kinematics more involved. In this case, the photon is produced via fragmentation of the final-state parton in a 2-to-2 process, $a b \rightarrow c d$. The momentum of the photon relative to the fragmenting parton is given by $z$, the lightcone momentum fraction of the heavy meson, and $\boldsymbol{k}_{\gamma}$, the transverse momentum of the meson with respect to the direction of the heavy quark. In a choice of coordinates where the fragmenting parton's momentum $p_{c}$ is along the $z$-axis, the photon's momentum can be written as

$$
p_{\gamma}=\left(E_{\gamma}, 0,0,\left|\boldsymbol{p}_{\gamma}-\boldsymbol{k}_{\gamma}\right|\right)+\left(0, \boldsymbol{k}_{\gamma}\right)
$$

where the first term on the right is the component along the fragmenting parton's direction and the second term is the component transverse to it. Here, $\boldsymbol{k}_{\gamma}$ is simply $\left(k_{\gamma_{x}}, k_{\gamma_{y}}, 0\right)=\left(\boldsymbol{k}_{\gamma_{\perp}}, 0\right)$. In the lab coordinates however, $\boldsymbol{k}_{\gamma}$ can have all three components nonzero and is specified as 
$\boldsymbol{k}_{\gamma}=k_{\gamma}(\sin \theta \cos \phi, \sin \theta \sin \phi, \cos \theta), \quad$ with $\left|\boldsymbol{k}_{\gamma}\right|=\left|\boldsymbol{k}_{\gamma_{\perp}}\right|$

and the orthogonality condition $\boldsymbol{k}_{\gamma} \cdot \boldsymbol{p}_{c}=0$ ensures that $\boldsymbol{k}_{\gamma}$ lies in a plane perpendicular to $\boldsymbol{p}_{c}$. The light-cone momentum fraction $z$ is given by

$$
z=\frac{p_{\gamma}^{+}}{p_{c}^{+}}=\frac{E_{\gamma}+\left|\boldsymbol{p}_{\gamma}-\boldsymbol{k}_{\gamma}\right|}{E_{c}+\left|\boldsymbol{p}_{c}\right|}=\frac{E_{\gamma}+\sqrt{\boldsymbol{p}_{\gamma}^{2}-\boldsymbol{k}_{\gamma}^{2}}}{2 E_{c}} .
$$

This gives us the expression for the energy of the heavy quark,

$$
E_{c}=\frac{E_{\gamma}+\sqrt{\boldsymbol{p}_{\gamma}^{2}-\boldsymbol{k}_{\gamma}^{2}}}{2 z} .
$$

The expression for $\boldsymbol{p}_{c}$ can be obtained from the fact that it is collinear with $\boldsymbol{p}_{\gamma}-\boldsymbol{k}_{\gamma}$ and that the unit vector constructed out of both must therefore be equal:

$\boldsymbol{p}_{c}=\frac{E_{c}}{E_{\gamma}-k_{\gamma}^{2}}\left(P_{T}-k_{\gamma} \sin \theta \cos \phi,-k_{\gamma} \sin \theta \sin \phi, P_{L}-k_{\gamma} \cos \theta\right)$

where we have used the orthogonality condition, $\boldsymbol{k}_{\gamma} \cdot \boldsymbol{p}_{c}=0$. Equations (A7) and (A8) relate the energy and momentum of the observed photon with that of the fragmenting parton for given values of $k_{\gamma}$ and $z$.

Using the expressions for the parton momenta given in Eqs. (A3) and (A8), one can solve the on-shell condition $\hat{s}+\hat{t}+\hat{u}=0$ for $z$ [14].

The term $d^{3} \boldsymbol{k}_{\gamma} \delta\left(\boldsymbol{k}_{\gamma} \cdot \hat{\boldsymbol{p}}_{c}\right)$ in Eqs. (9) and (10) ensures that the $\boldsymbol{k}_{\gamma}$ integration is only over momenta transverse to the fragmenting parton:

$$
\begin{aligned}
d^{2} \boldsymbol{k}_{\gamma_{\perp}} & =d^{3} \boldsymbol{k}_{\gamma} \delta\left(\boldsymbol{k}_{\gamma} \cdot \hat{\boldsymbol{p}}_{c}\right) \\
& =d k_{\gamma} k_{\gamma} d \theta d \phi \frac{\left|\boldsymbol{p}_{\gamma}-\boldsymbol{k}_{\gamma}\right|}{P_{T} \sin \phi_{1}}\left[\delta\left(\phi-\phi_{1}\right)+\delta\left(\phi-\left(2 \pi-\phi_{1}\right)\right)\right]
\end{aligned}
$$

where

$$
\cos \phi_{1}=\frac{k_{\gamma}-P_{L} \cos \theta}{P_{T} \sin \theta}
$$

Limits on $k_{\gamma}$ can be obtained by requiring $\left|\cos \phi_{1}\right| \leq 1$,

$$
\begin{aligned}
& \max {\left[P_{L} \cos \theta-P_{T} \sin \theta, 0\right] } \\
& \quad \leq k_{\gamma} \leq \max \left[P_{L} \cos \theta+P_{T} \sin \theta, 0\right] .
\end{aligned}
$$

[1] U. D’Alesio and F. Murgia, Prog. Part. Nucl. Phys. 61, 394 (2008).

[2] V. Barone, F. Bradamante, and A. Martin, Prog. Part. Nucl. Phys. 65, 267 (2010).

[3] C. Franco (COMPASS Collaboration), arXiv:1804.09516.

[4] X. d. Ji, J. P. Ma, and F. Yuan, Phys. Lett. B 597, 299 (2004).

[5] X. Ji, J. P. Ma, and F. Yuan, Phys. Rev. D 71, 034005 (2005).

[6] A. Bacchetta, D. Boer, M. Diehl, and P. J. Mulders, J. High Energy Phys. 08 (2008) 023.

[7] J. Collins, Cambridge Monogr. Part. Phys., Nucl. Phys., Cosmol. 32, 1 (2011).

[8] D. W. Sivers, Phys. Rev. D 41, 83 (1990).

[9] D. W. Sivers, Phys. Rev. D 43, 261 (1991).

[10] M. Anselmino, M. Boglione, U. D’Alesio, S. Melis, F. Murgia, E. R. Nocera, and A. Prokudin, Phys. Rev. D 83, 114019 (2011).

[11] M. Anselmino, M. Boglione, U. D'Alesio, E. Leader, S. Melis, F. Murgia, and A. Prokudin, Phys. Rev. D 86, 074032 (2012).

[12] M. Anselmino, M. Boglione, U. D’Alesio, S. Melis, F. Murgia, and A. Prokudin, Phys. Rev. D 88, 054023 (2013).

[13] M. Anselmino, M. Boglione, and F. Murgia, Phys. Lett. B 362, 164 (1995).
[14] U. D'Alesio and F. Murgia, Phys. Rev. D 70, 074009 (2004).

[15] M. Anselmino, M. Boglione, U. D’Alesio, E. Leader, S. Melis, and F. Murgia, Phys. Rev. D 73, 014020 (2006).

[16] L. Gamberg and Z. B. Kang, Phys. Lett. B 696, 109 (2011).

[17] U. D'Alesio, L. Gamberg, Z. B. Kang, F. Murgia, and C. Pisano, Phys. Lett. B 704, 637 (2011).

[18] U. D’Alesio, F. Murgia, and C. Pisano, Phys. Part. Nucl. 45, 676 (2014).

[19] U. D’Alesio, F. Murgia, C. Pisano, and P. Taels, Phys. Rev. D 96, 036011 (2017).

[20] A. Bacchetta, C. J. Bomhof, P. J. Mulders, and F. Pijlman, Phys. Rev. D 72, 034030 (2005).

[21] C. J. Bomhof, P. J. Mulders, and F. Pijlman, Eur. Phys. J. C 47, 147 (2006).

[22] U. D’Alesio, F. Murgia, and C. Pisano, J. High Energy Phys. 09 (2015) 119.

[23] C. Adolph et al. (COMPASS Collaboration), Phys. Lett. B 772, 854 (2017).

[24] M. Anselmino, M. Boglione, U. D’Alesio, E. Leader, and F. Murgia, Phys. Rev. D 70, 074025 (2004).

[25] R. M. Godbole, A. Kaushik, and A. Misra, Phys. Rev. D 94, 114022 (2016). 
[26] R. M. Godbole, A. Kaushik, and A. Misra, Phys. Rev. D 97, 076001 (2018).

[27] R. M. Godbole, A. Misra, A. Mukherjee, and V. S. Rawoot, Phys. Rev. D 85, 094013 (2012).

[28] R. M. Godbole, A. Misra, A. Mukherjee, and V. S. Rawoot, Phys. Rev. D 88, 014029 (2013).

[29] R. M. Godbole, A. Kaushik, A. Misra, and V. S. Rawoot, Phys. Rev. D 91, 014005 (2015).

[30] R. M. Godbole, A. Kaushik, A. Misra, V. Rawoot, and B. Sonawane, Phys. Rev. D 96, 096025 (2017).

[31] C. Aidala et al. (PHENIX Collaboration), Phys. Rev. D 98, 012006 (2018).

[32] J. G. Morfin and W. K. Tung, Z. Phys. C 52, 13 (1991).

[33] P. N. Harriman, A. D. Martin, W. J. Stirling, and R. G. Roberts, Phys. Rev. D 42, 798 (1990).

[34] M. Diemoz, F. Ferroni, E. Longo, and G. Martinelli, Z. Phys. C 39, 21 (1988).

[35] I. Schmidt, J. Soffer, and J. J. Yang, Phys. Lett. B 612, 258 (2005).

[36] Y. V. Kovchegov and M. D. Sievert, Phys. Rev. D 86, 034028 (2012); 86, 079906(E) (2012).

[37] M. Anselmino, M. Boglione, U. D’Alesio, A. Kotzinian, F. Murgia, and A. Prokudin, Phys. Rev. D 72, 094007 (2005); 72, 099903(E) (2005).
[38] M. Anselmino, M. Boglione, U. D’Alesio, A. Kotzinian, S. Melis, F. Murgia, A. Prokudin, and C. Turk, Eur. Phys. J. A 39, 89 (2009).

[39] M. Burkardt, Phys. Rev. D 69, 091501 (2004).

[40] J. C. Collins, Nucl. Phys. B396, 161 (1993).

[41] L. Gamberg and Z. B. Kang, Phys. Lett. B 718, 181 (2012).

[42] J. F. Owens, Rev. Mod. Phys. 59, 465 (1987).

[43] U. D’Alesio, F. Murgia, and C. Pisano, Phys. Rev. D 83, 034021 (2011).

[44] S. Kretzer, Phys. Rev. D 62, 054001 (2000).

[45] D. de Florian, R. Sassot, and M. Stratmann, Phys. Rev. D 75, 114010 (2007).

[46] A. Adare et al. (PHENIX Collaboration), Phys. Rev. D 90, 012006 (2014).

[47] J. Pumplin, D. R. Stump, J. Huston, H. L. Lai, P. M. Nadolsky, and W. K. Tung, J. High Energy Phys. 07 (2002) 012.

[48] L. Bourhis, M. Fontannaz, and J. P. Guillet, Eur. Phys. J. C 2, 529 (1998).

[49] A. D. Martin, R. G. Roberts, W. J. Stirling, and R. S. Thorne, Phys. Lett. B 531, 216 (2002).

[50] M. Glck, E. Reya, and A. Vogt, Eur. Phys. J. C 5, 461 (1998). 